\title{
Apports des isotopes naturels de l'eau à la caractérisation des mécanismes de recharge des aquifères du bassin de la Korama, Région de Zinder, Niger
}

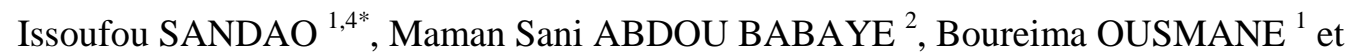 \\ Jean Luc MICHELOT ${ }^{3}$ \\ ${ }^{1}$ Université Abdou Moumouni, Faculté des Sciences et Techniques, Département de Géologie Niamey, \\ Niger. \\ ${ }^{2}$ Université Dan DickoDankoulodo, Faculté des Sciences et Techniques, UMR SERMUG, Département de \\ Géologie, BP:465, Maradi, Niger. \\ ${ }^{3}$ université Paris-Sud, Laboratoire d'hydrologie et de géochimie isotopique, UMR OrsayTerre CNRS, bât. \\ 504, 91405 Orsay, France. \\ ${ }^{4}$ Ministère de l'Hydraulique et de l'Assainissement, BP 2149 Niamey, Niger. \\ "Auteur correspondant ; E-mail: sandaoissoufou@gmail.com
}

\section{REMERCIEMENTS}

Les auteurs remercient vivement la Coopération Suisse au Niger et son Programme d'Hydraulique Rural et d'Appui au Secteur Eau et Assainissement (PHRASEA) pour son appui dans la conduite de cette étude.

\section{RESUME}

La présente étude porte sur les connaissances hydrochimiques et isotopiques des eaux souterraines du bassin de la Korama, situé en zone sahélienne où les nappes sont soumises à de fortes pressions démographiques et à des aléas de la pluviométrie. Cela se traduit par des fluctuations piézométriques saisonnières plus ou moins contrastées d'un secteur à un autre. Les besoins en prélèvements de ces ressources vont croissant, à cause de l'augmentation de la population et du développement des activités agricoles. Et pour optimiser l'exploitation de ces nappes et préserver l'équilibre écologique dans le bassin, il s'avère nécessaire de comprendre le fonctionnement hydrodynamique des aquifères en présence (origine et mécanisme de recharge des nappes). C'est ce qui fait l'objet de la présente étude hydrogéochimique. Trois campagnes (octobre et mars 2011, puis avril 2017) ont été ainsi organisées pour les prélèvements des échantillons des eaux des différentes nappes et la détermination des paramètres hydrochimiques (ions majeurs, Fluor, Nitrates) et isotopiques $\left({ }^{18} \mathrm{O}\right.$, ${ }^{2} \mathrm{H},{ }^{3} \mathrm{H},{ }^{14} \mathrm{C}$ et ${ }^{13} \mathrm{C}$ ). L'analyse des différents résultats hydrochimiques indique que les eaux souterraines du bassin de la Korama sont peu minéralisées. Elles sont essentiellement récentes pour la nappe phréatique des alluvions récentes, qui reçoit les eaux des précipitations avec des cachets d'évaporation. Pour les nappes plus profondes des grès de Mallawa, les eaux sont anciennes, car rechargées à partir des précipitations ayant intervenu au cours des paléoclimats plus humides que le climat actuel, c'est-à-dire durant la période comprise entre 12000 et 10000 ans BP. Cependant, les différentes nappes sont en intercommunication hydraulique, et il existerait des échanges d'eau plus ou moins importants selon les périodes de l'année (basses ou hautes eaux). (C) 2018 International Formulae Group. All rights reserved.

Mots clés : hydrochimie, isotopes, recharge nappe, Korama, Zinder, Niger. 


\title{
Contribution of natural isotopes of water to the characterization of recharge of Korama valley aquifer, region of Zinder in Niger
}

\begin{abstract}
This study focuses on the hydrochemical and isotopic parameters in groundwater basin of Korama, in a context of Sahelian climate and where the aquifers are subjected to high population pressure and erratic rainfall. This resultsin seasonal groundwater levels fluctuations which are very contrasting from one sector to the other. In view of the above mentioned pressure on groundwater resources, it becomes important to conduct studies that enable a better understanding of the functioning of hydrodinamique Basin aquifers (origin and mechanism of groundwater recharge). Three (3) sampling water campaigns were conducted on the three aquifers for hydrochemical (majors ion, Fluoride , Nitrates ) and isotopics $\left({ }^{18} \mathrm{O},{ }^{2} \mathrm{H},{ }^{3} \mathrm{H},{ }^{14} \mathrm{C}\right.$, and ${ }^{13} \mathrm{C}$ ) analyzes. The results showed that the groundwater of the Korama basin are of low mineralization. They are essentially modern for the recent alluvial aquifer which receives water from rainfall, which is characterized by evaporated samples. For other deeper aquifers, the waters are older because they are recharged from snow that occurred during the paleoclimate between 12000 and 10000 years BP that is more humid than the current climate. However, the different aquifers are inter-connected and there exists exchange of water by drainage, which can be important depending on the period of the years.
\end{abstract}

(C) 2018 International Formulae Group. All rights reserved.

Keywords: hydrochimy, isotops, recharge, korama, Zinder Niger.

\section{INTRODUCTION}

Le bassin de la Korama est une zone humide où l'agriculture irriguée se développe à partir des eaux des nappes de faibles profondeurs. Seulement, les fluctuations des niveaux statiques y sont importantes à cause, non seulement des prélèvements, mais aussi des variations des paramètres hydroclimatiques (Sandao, 2010; Soumaila, 2016).

Cette étude a été entreprise pour mieux caractériser la dynamique et les origines des recharges de ces nappes très sensibles et sous fortes pressions naturelles et anthropiques. Sa finalité est de permettre une optimisation des prélèvements et une préservation des écosystèmes du bassin.

La méthode utilisée est celle du traçage chimique et isotopique de plus en plus utilisée en hydrogéologie pour étudier les comportements des nappes d'eau souterraines, (Goni, 2006).

Ainsi, trois (3) campagnes de prélèvement d'échantillons d'eau ont été organisées en octobre et mars 2011, puis en avril 2017. Les résultats analytiques des paramètres chimiques (ions majeurs, fluor, nitrates) et isotopiques $\left({ }^{18} \mathrm{O},{ }^{2} \mathrm{H},{ }^{3} \mathrm{H},{ }^{14} \mathrm{C}\right.$ et ${ }^{13} \mathrm{C}$ ) ont été interprétés et discutés.

Cela a permis de déterminer l'origine des eaux des différentes nappes, de comprendre les mécanismes de leur renouvellement et d'estimer l'importance des éventuels échanges entre la nappe phréatique superficielle des alluvions récentes et les nappes plus profondes des aquifères du socle précambrien granitique ou quartzitique, des grès du Quaternaire ancien, des grès Crétacé et Tertiaire du Continental Hamadien et des grès du Continental Terminal.

\section{MATERIEL ET METHODES}

Présentation de la zone d'étude

La zone d'étude ou bassin de la Korama correspond à l'extrême Ouest du grand bassin du Lac Tchad et se situe aux confins du Niger et du Nigéria (Figure 1). Pour une superficie de $120 \times 100 \mathrm{~km}^{2}$, le bassin est allongé est-ouest. Il est limité au Nord et à l'Est par les affleurements des granites du socle précambrien de Damagaram Mounio, à l'Ouest par le bassin occidental du Niger (bassin des Iullemmeden) et au Sud par la frontière du Niger avec le Nigéria (Sandao, 2013). 
Avec une densité moyenne de 110 $\mathrm{hbts} / \mathrm{km}^{2}$ (INS, 2012), c'est l'une des régions les plus peuplées du Niger. Les activités économiques y sont l'agriculture pluviale, l'élevage et, de plus en plus, l'agriculture irriguée à partir des eaux souterraines peu profondes.

Le climat est de type sahélien, avec une courte saison de pluies de quatre mois, de juin à septembre, et une longue saison sèche d'environ huit mois, d'octobre à mai. Les hauteurs pluviométriques moyennes annuelles augmentent du Nord au Sud, avec $500 \mathrm{~mm}$ à la station synoptique de Zinder aéroport, au Nord du bassin, et plus de $600 \mathrm{~mm}$ à la station de Magaria au Sud du bassin (DMN Niger, 2011). Ces précipitations se caractérisent par une grande variabilité spatio-temporelle.

C'est un bassin endoréique où le réseau hydrographique est bien structuré, et correspond à la rivière Korama et ses affluents. Ces cours d'eau sont en voix de fossilisation et ne sont représentés que par des chapelets des mares par endroit.

\section{Contexte géologique de la zone d'étude}

Dans le bassin de la Korama, les formations sédimentaires reposant sur le socle précambrien granitique et quartzique de Damagaram Mounio, sont de bas en haut, selon (Greigert, 1972) (Figure 2) :

- les grès argileux ferrugineux du Continental Intercalaire / Hamadien, du Crétacé inférieur, dans la partie nordouest;

- les grès argileux du Tertiaire (Continental Terminal) au sud-ouest;

- les grés argileux fins de couleur rose du Quaternaire ancien, dits grès de Mallawa, au sud et sud-est ;

- les sables alluvionnaires, moyens à grossiers du Quaternaire, appelés dépôts des sables récents, au centre du bassin.

\section{Contexte hydrogéologique de la zone}

Les principaux aquifères du bassin de la Korama sont de bas en haut (Sogetha, 1964):
- les aquifères discontinus du socle cristallophyllien et cristallin ;

- l'aquifère des grès argileux à ciment ferrugineux du Continental Intercalaire / Hamadien ;

- l'aquifère des grès argileux du Continental Terminal ;

- l'aquifère des grés fins et argileux du Quaternaire ancien ou grès de Mallawa ;

- l'aquifère des sables alluvionnaires récents, plus important en étendu et renfermant l'essentiel des réserves en eau mobilisables du bassin.

La profondeur des niveaux statiques dans ces réservoirs varie de 0 à 10 mètres dans la nappe alluvionnaire des sables alluvionnaires récents et de 15 à plus de 30 mètres dans les aquifères du socle, des grès argileux du Continental Hamadien et Terminal et des grès de Mallawa.

\section{Méthodologie}

\section{Outils et données utilisés}

La mise en œuvre de cette étude a nécessité l'application de différents outils pour la collecte de données sur le terrain et leurs analyses. Pour les données, il s'agit surtout :

- des données des campagnes de suivis piézométriques, à travers un réseau optimisé de suivi, constitué de 53 piézomètres indiqués sur la carte de la Figure 1 (Sandao, 2010). Ce réseau est constitué des puits et des forages existants. Pour assurer un bon maillage, des piézomètres complémentaires ont été réalisés. Les mesures des niveaux d'eau ont été effectuées avec une sonde piézométrique manuelle. Après le nivellement de tous les points de mesures, ces niveaux d'eau sont transformés en altitude piézométrique.

- des données d'analyses des éléments chimiques des échantillons d'eau souterraine, prélevés au cours des trois (3) campagnes d'échantillonnage. La première a permis de prélever 36 échantillons en périodes des basses eaux (mars 2011), la seconde pour 42 échantillons, en octobre 2011 et la 
dernière en avril 2017 pour 42 échantillons. Les points d'eau échantillonnés sont représentés sur la carte de la Figure 3 ; ces analyses ont été effectuées avec des spectromètres DR et des spectrophotomètres à flame ;

- des données d'analyes des isotopes $\left(\boldsymbol{\delta}^{18} \mathrm{O}\right.$, $\delta^{2} \mathrm{H},{ }^{3} \mathrm{H},{ }^{13} \mathrm{C}$ et ${ }^{14} \mathrm{C}$ ) des échantillons des eaux souterraines, prélevés au cours des mêmes campagnes d'échantillonnage et sur les mêmes ouvrages. Les analyses du couple oxygène-18 / deutérium ont été réalisés par le Laboratoire IDES de l'Université de Paris-Sud (Orsay) et celles relatives au tritium, au laboratoire HYDROISOTOP de Schweitenkirchen en Allemagne. Les résultats des analyses sont exprimés en $\delta \%$ vs V-SMOW. Les erreurs analytiques sont respectivement de $\pm 0,2 \delta \%$ et $\pm 2 \delta \%$ pour l'oxygène- 18 et le deutérium. S'agissant des teneurs en tritium exprimées en unité de tritium (UT), la précision est de \pm 1 .

- des données de mesure in situ des paramètres physiques des eaux, lors des missions de suivis qualitatif et quantitatif. Les mesures de ces paramètres sont directement effectuées sur le terrain avec un multiparamètre.

- des données d'analyses hydrochimiques (ions majeurs) et isotopiques $\left(\boldsymbol{\delta}^{18} \mathrm{O}\right.$ et $\left.\boldsymbol{\delta}^{2} \mathrm{H}\right)$ de 8 échantillons d'eaux de précipitations, collectés au cours de la saison pluvieuse de 2011 au niveau de la station pluviométrique de Zinder..

Les quatre (4) laboratoires pour les analyses et contre-analyses chimiques, et les analyses isotopiques, sont :

- le laboratoire d'analyse des eaux de la Direction Régionale de l'Hydraulique de Zinder, pour les éléments chimiques ;

- le laboratoire des eaux du Département de Géologie de l'Université Abdou Moumouni de Niameypour les éléments chimiques ;

- le laboratoire d'Hydrologie et de Géochimie isotopique de l'Université Paris Sud, Orsay (France), pour les isotopes $\boldsymbol{\delta}^{18} \mathrm{O}, \boldsymbol{\delta}^{2} \mathrm{H},{ }^{13} \mathrm{C}$ et ${ }^{14} \mathrm{C}$.
- le laboratoire HYDROISOTOP gmbh, en Allemagne, pour le tritium $\left({ }^{3} \mathrm{H}\right)$.

Pour le traitement des données, divers outils informatiques ont été appliqués dont le logiciel ArcGis 9.3 pour la cartographie, le diagramme pour l'hydrochimie et le tableur excel pour les autres traitements.

\section{Méthodes}

Les prélèvements des échantillons ont été faits sur la base de la configuration hydrogéologique et ont concerné toutes les nappes en présence. Tous les sites sont géoréférencés et reportés sur un fond cartographique (Figure 3), (Guillaume et Christian, 2000).

Sur chaque site, les paramètres physico-chimiques (conductivité, température, $\mathrm{pH}$ et alcalinité) ont été mesurés in situ, avant de procéder au rinçage et remplissage des flacons plastiques en polyéthylène de $500 \mathrm{ml}$ pour les analyses chimiques. Des pullulés en verre bronzé de $30 \mathrm{ml}$ et $15 \mathrm{ml}$ ont été utilisés pour les prélèvements des échantillons destinés au dosage de $\mathrm{l}^{18} \mathrm{O}$ et du ${ }^{2} \mathrm{H}$.

Les éléments chimiques ont été dosés par titrimétrie $(\mathrm{Ca}$ et $\mathrm{Mg})$, spectrométrie $\left(\mathrm{Fe}^{2+}, \mathrm{Fe}^{3+}, \mathrm{NO}_{3}^{-}, \mathrm{NO}_{2}^{--}, \mathrm{F}, \mathrm{SO}_{4}^{--}, \mathrm{Cl}^{-}\right)$et photométrie à flamme ( $\mathrm{Na}$ et $\mathrm{K})$. Les résultats sont validés par la méthode de calcul de la balance ionique, avec une erreur de $\pm 5 \%$.

Les isotopes ${ }^{18} \mathrm{O}$ et ${ }^{2} \mathrm{H}$ ont été dosés par spectrométrie de masse après équilibration de l'échantillon avec $\mathrm{CO}_{2}$ pour $1^{118} \mathrm{O}$ et avec l'hydrogène pour le ${ }^{2} \mathrm{H}$. Les marges d'erreur admises sont de $\pm 0,2 \%$ SMOW.

Le tritium a été dosé par la Spectrométrie de scintillation en phase liquide (LSC) après concentration par électrolyse. Les teneurs en ${ }^{3} \mathrm{H}$ sont exprimées en unités de tritium (TU) avec une erreur de \pm 1 UT.

Les activités en ${ }^{14} \mathrm{C}$ et les teneurs en ${ }^{13} \mathrm{C}$ ont été déterminées par spectrométrie de masse, après piégeage et extraction du $\mathrm{CO}_{2} \mathrm{de}$ l'échantillon avec de l'acide phosphorique.

Les nombres des échantillons prélevés et les différents paramètres analysés sont indiqués dans le Tableau 1. 


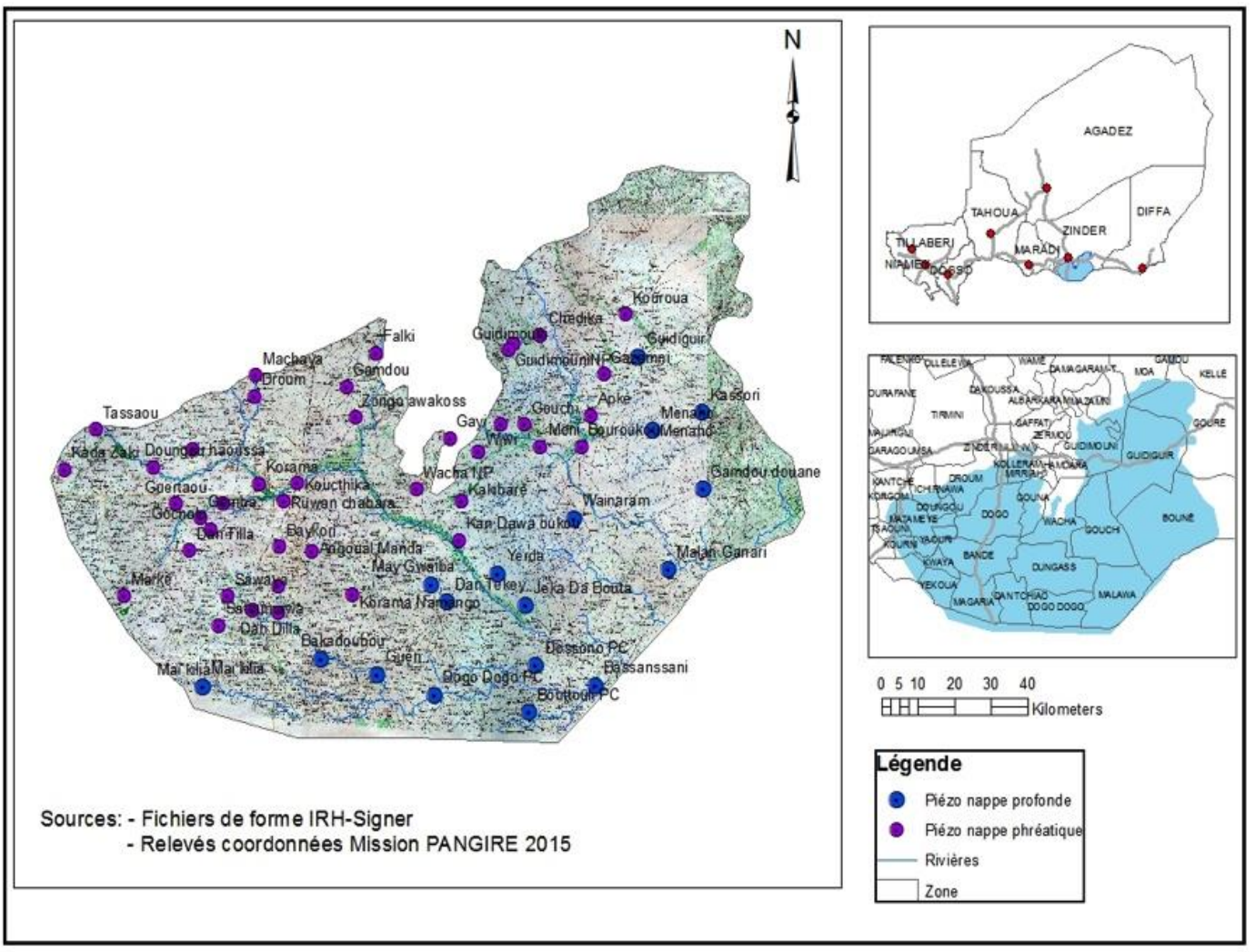

Figure 1: Localisation et présentation de la zone d'étude.

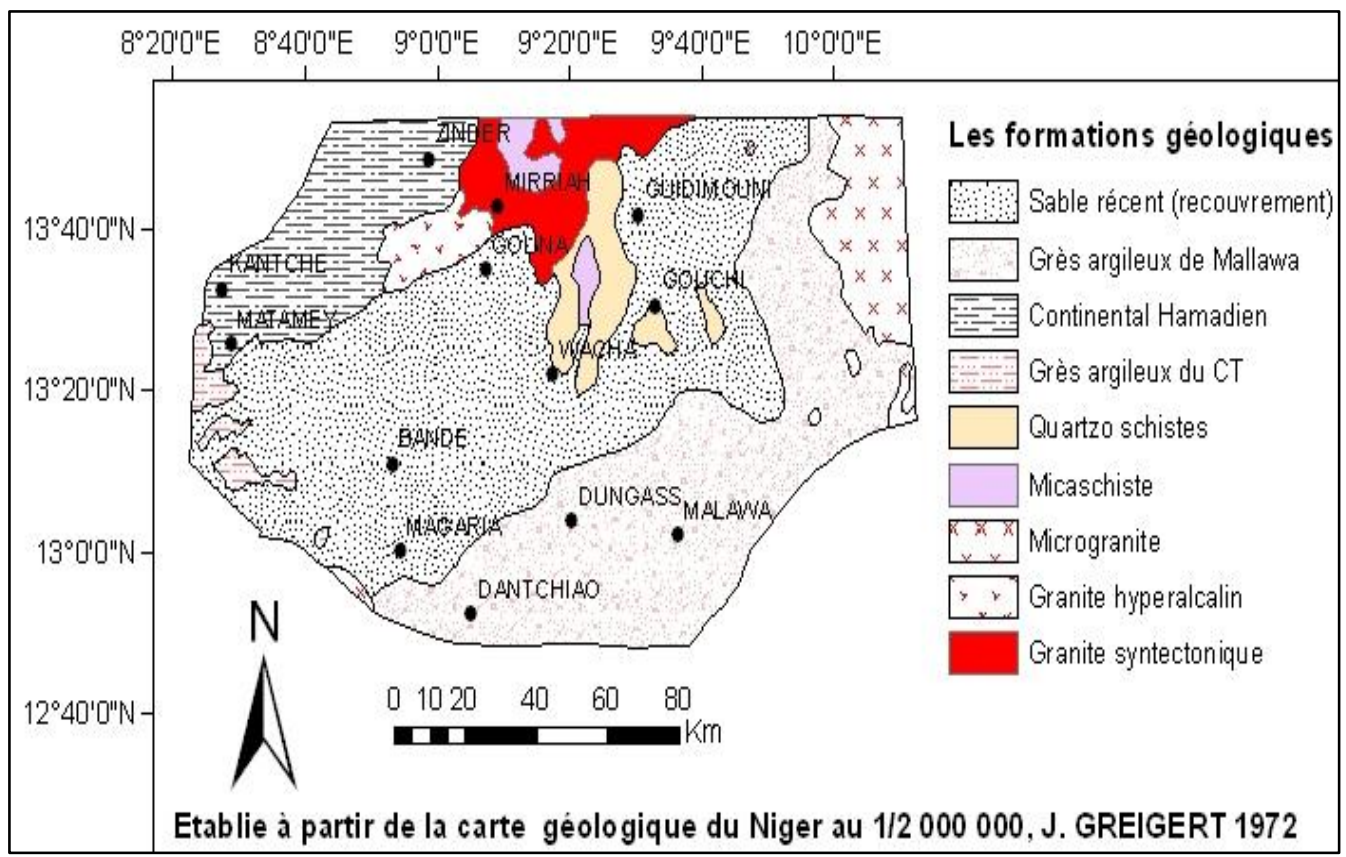

Figure 2 : Carte géologique simplifiée du bassin de la Korama. 


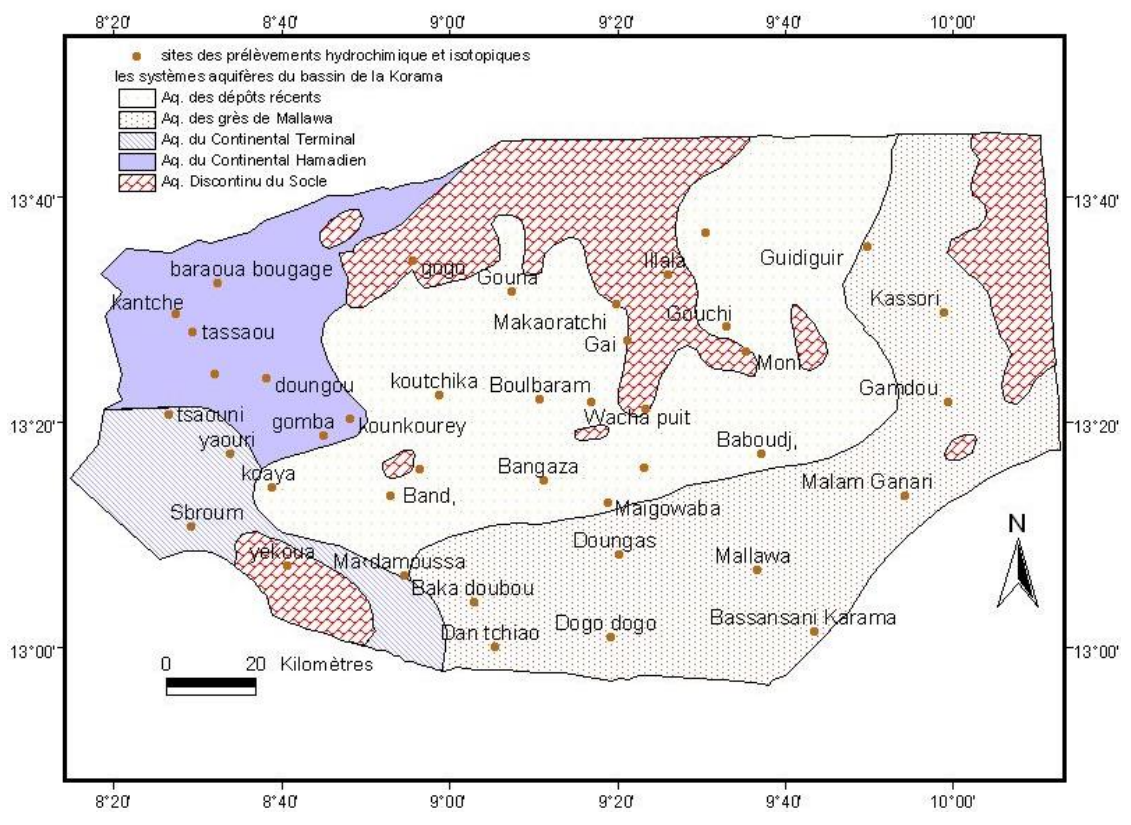

Figure 3: Carte de répartition des sites d'échantillonnage pour les analyses chimiques et isotopiques.

Tableau 1 : Nombre d'échantillons et paramètres analysés par campagne.

\begin{tabular}{lcccc}
\hline Périodes des prélèvements & $\begin{array}{c}\text { Eléments } \\
\text { chimiques }\end{array}$ & $\begin{array}{c}\text { Isotopes } \\
{ }^{\mathbf{1 8}} \mathbf{O} \text { et }{ }^{\mathbf{2}} \mathbf{H}\end{array}$ & $\begin{array}{c}\text { Isotope } \\
{ }^{\mathbf{3}} \mathbf{H}\end{array}$ & $\begin{array}{c}\text { Isotopes } \\
\mathbf{1 3}^{\mathbf{1 3}} \mathbf{C} \text { et }{ }^{\mathbf{1 4}} \mathbf{C}\end{array}$ \\
\hline $1^{\text {ère }}$ campagne, octobre 2011 & 37 & 29 & 4 & \\
$2^{\text {ème }}$ campagne, mars 2017 & 42 & 42 & & 4 \\
$3^{\text {ème }}$ campagne, avril 2017 & 42 & 42 & & \\
\hline
\end{tabular}

\section{RESULTATS}

\section{Caractéristiques piézométriques de la zone d'étude}

La carte piézométrique établie en janvier 2016 (Figure 4), montre que tous les niveaux s'intègrent dans un seul système piézométrique à l'échelle du bassin, indiquant ainsi des échanges d'eau entre les différentes unités.

L'écoulement général des eaux est orienté ouest-est, et fait apparaître 3 dômes piézométriques (au Nord-est, au centre et au Sud-ouest), qui représentent les principales aires de recharge des nappes. La dépression piézométrique au Sud-est, dans les grès argileux de Mallawa, constitue l'exutoire des eaux souterraines du bassin.

Au cours de la période de suivi (2009 à 2017), les fluctuations saisonnières des niveaux statiques sont nulles à très faibles (quelques centimètres seulement) dans les nappes des grès de Mallawa (Sud-est) et des grès du Continental Hamadien (Nord-ouest). On pourrait ainsi, supposer que ces nappes ne se seraient pas du tout rechargées ou alors, les apports annuels seraient équivalents aux prélèvements.

Par contre, dans le Centre et le Sudouest $\mathrm{du}$ bassin, les fluctuations piézométriques annuelles varient de 0,5 à $1 \mathrm{~m}$ dans les nappes des grès du Continental 
Terminal et celles des sables alluvionnaires. Ces variations piézométriques pourraient être dues, en partie, à l'infiltration saisonnière des précipitations à travers les sables filtrants de l'aquifère des alluvions récentes.

\section{Paramètres physiques des eaux souterraines.}

Les paramètres physiques des eaux sont donnés dans les Tableaux 2 et 3 .

Les valeurs de la conductivité mesurées sont faibles (plus de 95\% sont inférieures à $500 \mu \mathrm{S} / \mathrm{cm}$ ). Elles augmentent pendant la saison des pluies, particulièrement dans la nappe des alluvions récentes où les niveaux statiques sont proches de la surface du sol.

Les $\mathrm{pH}$ des eaux souterraines dans la zone d'étude sont en général acides, avec deux pôles de regroupement: les valeurs comprises entre 6 et 7 unités $\mathrm{pH}$, dans les nappes phréatiques du Quaternaire (alluvions et grès argileux de Mallawa), et les valeurs plus faibles, inférieures à 6 unités $\mathrm{pH}$, au niveau des nappes du Crétacé (grès argileux sidérolitique du Continental Intercalaire/Hamadien) et du Tertiaire (grès argileux du Continental Terminal).

Les valeurs moyennes des températures des eaux sont faibles $\left(26,5^{\circ} \mathrm{C}\right.$ en octobre et $29,9{ }^{\circ} \mathrm{C}$ en mars), et semblent suivre le régime climatique de la zone, car proches des valeurs moyennes atmosphériques. Elles sont également en corrélation avec la profondeur des nappes.

\section{Paramètres chimiques des eaux des nappes}

Les paramètres chimiques des eaux des nappes de la zone sont donnés dans les Tableaux 2 et 3. Pour la campagne d'octobre 2011, mis à part un seul échantillon (Kounkourey), excessivement chargé à cause de la pollution anthropique, la minéralisation totale des eaux varie de 0.64 à $12,71 \mathrm{meq} / \mathrm{l}$, avec une moyenne de 3,58 meq/l. Aussi, pour 32 des 42 échantillons, soit $76 \%$, la somme totale des éléments chimiques ne dépasse-telle pas $5 \mathrm{meq} / \mathrm{l}$.

En mars 2011, et dans les mêmes conditions, la minéralisation totale varie de
0,68 à 17,04 meq/l avec une moyenne de 3,87 meq $/ 1.75 \%$ des échantillons pour cette campagne, ont une minéralisation totale inférieure à $5 \mathrm{meq} / \mathrm{l}$. Les résultats de la campagne d'avril 2017 confirment la même situation de mars. Ainsi, on relève que les eaux souterraines du bassin de la Korama sont très peu minéralisées, quelle que soit la période de l'année. En effet, les valeurs de la minéralisation totale obtenues sont faibles et inférieures aux moyennes trouvées dans les nappes phréatiques de la bande Sahélienne et dans les zones des Dallols, proches de la zone d'étude (Guéro, 2003). La répartition spatiale de la minéralisation ne semble pas indiquer de contraste entre les différentes nappes du bassin, les eaux sont dans la même gamme de charge ionique.

Les résultats analytiques de la période des basses eaux, (en mars 2011), reportés dans le diagramme de Piper (Figure 5), montrent que le faciès chimique des eaux est du type bicarbonaté calcique dans les nappes du socle granitique, des grès argileux de Mallawa et des grès du Continental Hamadien. Ce faciès pourrait être plus lié à une minéralisation par interaction entre l'eau et les matériaux géologiques des aquifères et moins à l'infiltration des eaux atmosphériques. Ce qui confirme l'hypothèse des faibles apports des précipitations dans la recharge de ces nappes. Par contre la nappe phréatique des dépôts récents affiche un hydro faciès de type chloruré-sulfaté calcique avec parfois une tendance nitratée. Ce cachet différent qui indiquerait un processus d'acquisition des ions différent avec celui des autres nappes.

\section{Teneurs en isotopes stables de l'eau : oxygène-18 et deutérium}

Les résultats des analyses des isotopes ${ }^{18} \mathrm{O}$ et ${ }^{2} \mathrm{H}$ sur les échantillons des 2 campagnes, ainsi que les valeurs des excès en deutérium (" $\mathrm{d} "=\delta^{2} \mathrm{H}-8 \mathrm{x} \delta^{18} \mathrm{O}$ ), sont présentés dans les Tableaux 4 et 5 . Sur les échantillons de la nappe phréatique des alluvions récentes, les teneurs en $\delta^{18} \mathrm{O}$ varient de $-4,98$ à $-1,98 \%$ SMOW, avec une moyenne de $-3,90 \%$ SMOW en mars 2011, et de $-4,49$ à $-1,65 \%$ SMOW, avec une moyenne de - 
3,81\% SMOW en octobre 2011. Les teneurs en deutérium $\left(\delta^{2} \mathrm{H}\right)$ pour les mêmes échantillons, s'inscrivent entre $-2,14$ et $18,73 \%$ SMOW, avec une moyenne de $27,14 \%$ SMOW en mars 2011, et entre $-30,41$ et $-12,20 \%$ SMOW, avec une moyenne de $26,80 \%$ SMOW en octobre 2011. Ces valeurs sont très proches de celles trouvées dans des nappes phréatiques de la zone sahélienne en général et du Niger en particulier (Taupin, 1994 ; Guéro, 2003 ; Abdou, 2012).On note une augmentation des valeurs en $\delta^{18} \mathrm{O}$ et $\delta^{2} \mathrm{H}$ au cours de la saison de pluie. Ces valeurs, sont supérieures à celles caractéristiques des précipitations actuelles dans la sous-région, 4,6 à $-4 \%$ SMOW pour ${ }^{18} \mathrm{O}$ (Ousmane, 1988 ; Zakara et al., 1993; Taupin, 1994 ; Guéro, 2003 ; Sandao, 2013).

En ce qui concerne les autres nappes plus profondes de la zone d'étude, en mars 2011 , les résultats globaux des teneurs en ${ }^{18} \mathrm{O}$ se situent entre $-5,95$ et $-3,40 \%$ SMOW, avec une moyenne de $-4,54 \%$ SMOW. Les teneurs en $\delta^{2} \mathrm{H}$ sont comprises entre $-42,05$ et $23,16 \%$ SMOW, avec une moyenne de $31,10 \%$ SMOW. Pour les prélèvements d'octobre 2011, les teneurs en $\delta^{18} \mathrm{O}$ varient de $-5,87$ à $-3,63 \%$ oMOW, avec une moyenne de $-4,76 \%$ SMOW, les teneurs en $\delta^{2} \mathrm{H}$ sont comprises entre $-41,46$ et $-26,07 \%$ SMOW, avec une moyenne de $-32,80 \%$ SMOW. Les valeurs en ${ }^{18} \mathrm{O}$ et ${ }^{2} \mathrm{H}$ au niveau de ces nappes plus profondes sont inférieures à celles des précipitations, et restent relativement stables dans le temps. Toutefois, elles sont plus élevées que celles trouvées dans les mêmes nappes, plus profondes et en charge ? Sous le Continental Intercalaire et le Continental Terminal, mais dans leur portions situées plus à l'ouest, dans les régions de Tahoua et Dosso, où les teneurs en ${ }^{18} \mathrm{O}$ varient entre $7,9 \%$ et $-7,6 \%$ SMOW et les teneurs en ${ }^{2} \mathrm{H}$ entre -57,6 et 56,4\%o SMOW (Guéro, 2003).

\section{Teneurs en Tritium ${ }^{3} \mathrm{H}$ et ${ }^{13} \mathrm{C}$ et activités en ${ }^{14} \mathrm{C}$}

Huit (8) échantillons des eaux souterraines des différentes nappes de la zone ont été prélevés en mars et octobre 2011 pour la détermination des teneurs en tritium. Les résultats analytiques du tritium sont indiqués dans le Tableau 6.

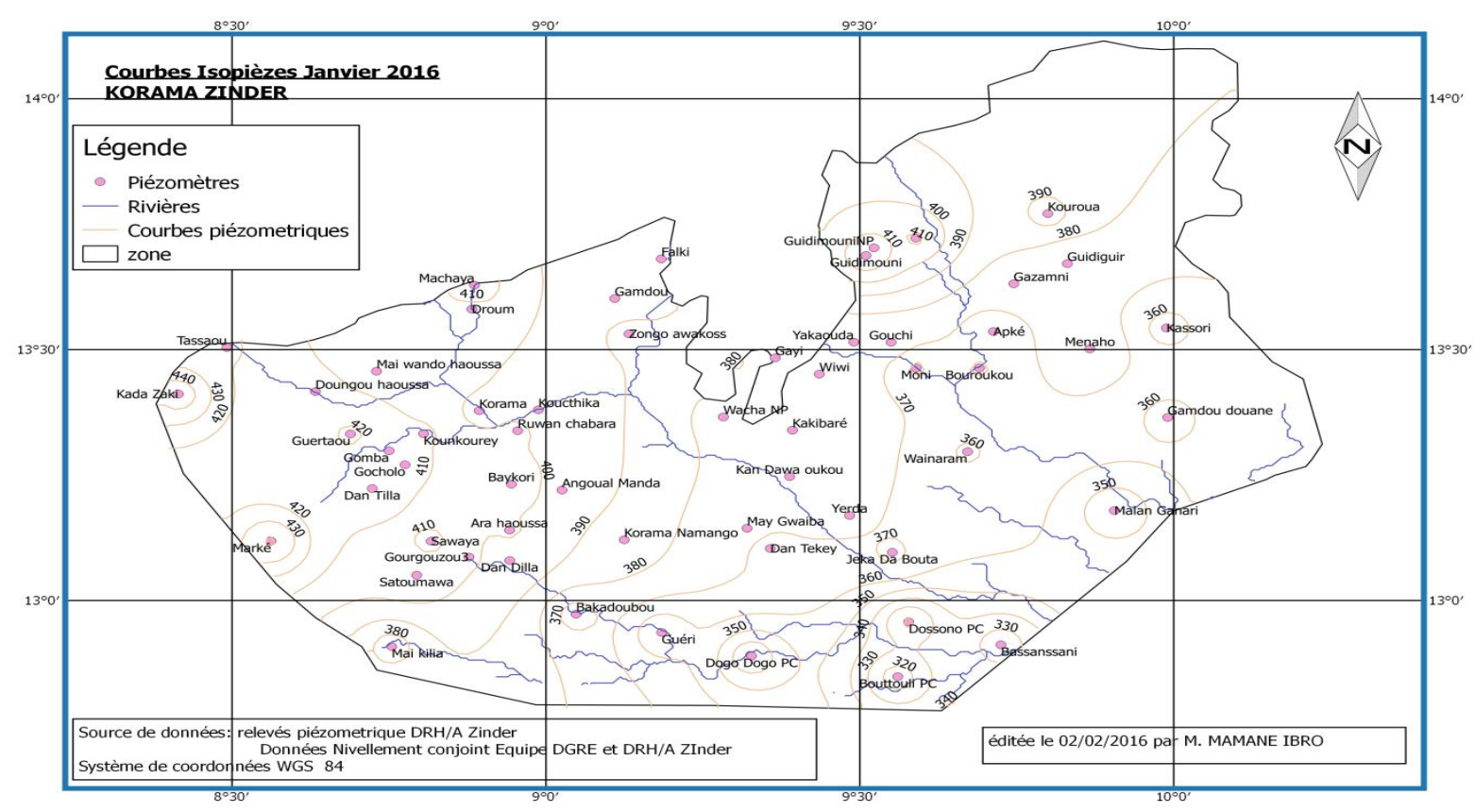

Figure 4: Carte piézométrique du bassin de la Korama en janvier 2016. 
Tableau 2 : Résultats des analyses chimiques des échantillons des eaux souterraines, prélevés au cours de la campagne d'échantillonnage de mars 2011.

\begin{tabular}{|c|c|c|c|c|c|c|c|c|c|c|c|c|c|c|c|c|c|c|}
\hline \multirow{3}{*}{$\mathbf{N}^{\circ}$} & \multirow[b]{3}{*}{ Localité } & \multirow[b]{3}{*}{$\begin{array}{l}\text { Type } \\
\text { PEM }\end{array}$} & \multirow[b]{3}{*}{ Latitude } & \multirow[b]{3}{*}{ Longitude } & \multirow[b]{3}{*}{ NS } & \multirow{2}{*}{\multicolumn{3}{|c|}{ Paramètres physiques }} & \multirow{2}{*}{\multicolumn{10}{|c|}{ Paramètres chimiques en mg/l, prélèvements de mars 2011}} \\
\hline & & & & & & & & & & & & & & & & & & \\
\hline & & & & & & $\begin{array}{l}\mathrm{C} \\
(\mathrm{ms} / \mathrm{cm})\end{array}$ & $\mathbf{p H}$ & $\mathbf{T}\left({ }^{\circ} \mathbf{C}\right)$ & $\mathbf{N a}$ & $\mathbf{K}$ & $\mathbf{C a}$ & Mg & $\mathbf{F e}$ & $\mathrm{HCO3}$ & $\mathbf{C l}$ & SO4 & NO3 & $\mathbf{F}$ \\
\hline \multicolumn{19}{|c|}{ Echantillons de la nappe phréatique des dépôts récents } \\
\hline 1 & Guidimouni & forage & 13,69194 & 9,50944 & 4,5 & 374 & 6,90 & 30,10 & 28 & 28 & 34,4 & 10,5 & 0,11 & 97,6 & 40 & 21 & 53,68 & 0,22 \\
\hline 2 & $\begin{array}{l}\text { Guidimouni } \\
\text { AEP }\end{array}$ & forage & 13,69994 & 9,50944 & 22 & 74 & 6,30 & 32,00 & 2 & 4 & 5,6 & 1,92 & 0,05 & 22,5 & 3 & 0 & 15,4 & 0,08 \\
\hline 3 & Maigowaba & puit & 13,16667 & 9,31556 & 3,34 & 163 & 7,30 & 27,20 & 4 & 4,5 & 18,4 & 3,84 & 0,05 & 42,7 & 7 & 4 & 32,12 & 0,55 \\
\hline 5 & Kandawaoukou & forage & 13,23556 & 9,38833 & 1,74 & 246 & 6,56 & 31,00 & 6,5 & 4 & 27,2 & 1,46 & 0 & 7,93 & 24,1 & 5 & 55 & 0 \\
\hline 6 & Kakibaré & forage & 13,35056 & 9,39000 & 2,00 & 116 & 5,90 & 31,20 & 17 & 2 & 2,8 & 0,243 & 0,13 & 26,5 & 6,2 & 4 & 18,92 & 0,19 \\
\hline 7 & Wachapuit & puit & 13,36583 & 9,28256 & 10,00 & 505 & 6,85 & 30,40 & 35 & 25 & 20 & 7,2 & 0,05 & 54,9 & 50 & 26 & 37,84 & 0,02 \\
\hline 8 & Boulbaram & forage & 13,37000 & 9,18000 & 5,50 & 198 & 6,70 & 30,90 & 7 & 3 & 12 & 8,16 & 0,28 & 48,8 & 11 & 23 & 14,5 & 0,7 \\
\hline 9 & Tassaou & forage & 13,50417 & 8,49194 & 5,00 & 117 & 6,02 & 28,40 & 9 & 2 & 8,8 & 1,82 & 0,35 & 34,16 & 10 & 2 & 21,5 & 0,15 \\
\hline 10 & Doungou & forage & 13,41083 & 8,63778 & 1,70 & 77 & 5,74 & 30,00 & 1 & 0 & 13,6 & 2,43 & 0,03 & 15,5 & 1,8 & 0 & 34,76 & 0,1 \\
\hline 11 & Kounkourey & puit & 13,33222 & 8,80500 & 3,24 & 1950 & 5,60 & 28,26 & 170 & 105 & 114 & 52,97 & 0,12 & 518,5 & 142 & 320 & 83,6 & 0,32 \\
\hline 12 & Gomba haoussa & puit & 13,29861 & 8,75000 & 5,66 & 701 & 6,82 & 29,10 & 5 & 6 & 78 & 11,42 & 0,23 & 115,9 & 44 & 40 & 62,04 & 0,11 \\
\hline 14 & Koutchika & puit & 13,37778 & 8,98167 & 1,05 & 385 & 6,78 & 29,50 & 12,4 & 8 & 34,8 & 8,24 & 0,34 & 108 & 12,4 & 54 & 4,4 & 0,31 \\
\hline 15 & Gogo & puit & 13,64306 & 8,92833 & 3,00 & 157 & 6,61 & 28,50 & 8 & 6 & 17,6 & 0,5 & 0,87 & 48,8 & 7 & 8 & 23,76 & 0,17 \\
\hline 16 & Maïdamoussa & forage & 13,02222 & 8,91083 & 15,00 & 54 & 5,40 & 31,00 & 3 & 2 & 14,4 & 1,92 & 0,02 & 48,8 & 4 & 1 & 11,4 & 0,2 \\
\hline
\end{tabular}


I. SANDAO et al. / Int. J. Biol. Chem. Sci. 12(4): 1931-1954, 2018

\begin{tabular}{|c|c|c|c|c|c|c|c|c|c|c|c|c|c|c|c|c|c|}
\hline Bandé puit & puit & 13,17944 & 8,88417 & 13,73 & 27 & 5,90 & 30,00 & 3 & 1 & 2,4 & 0,5 & 0,09 & 12,2 & 4 & 0 & 6 & 0,2 \\
\hline Beykori pc & puit & 13,23056 & 8,94111 & 7,18 & 164 & 6,53 & 30,00 & 9 & 8 & 12 & 3,36 & 0,07 & 48,8 & 14 & 5 & 9,68 & 0,08 \\
\hline
\end{tabular}

Echantillons des nappes profondes de socle, des grès argileux de Mallawa, des grès du Continental Hamadien et des grès du Continental Terminal

\begin{tabular}{|c|c|c|c|c|c|c|c|c|c|c|c|c|c|c|c|c|c|}
\hline Baraouabougage & forage & 13,59876 & 8,54004 & 40,00 & 143 & 6,50 & 29,00 & 6,5 & 5 & 22,4 & 3,5 & 0,12 & 97,6 & 4 & 4 & 3 & 0,49 \\
\hline Kantché pc & puit & 13,53917 & 8,45889 & 13,96 & 183 & 6,53 & 29,00 & 11 & 11 & 20,8 & 3,82 & 0,09 & 97,6 & 7,5 & 2 & 10 & 0,35 \\
\hline Dan tchiao & forage & 12,88028 & 9,09278 & 21,45 & 41 & 6,30 & 31,00 & 2 & 1,25 & 5,6 & 1,92 & trace & 33,5 & 3 & 0 & 3,08 & 0,62 \\
\hline Doungas & forage & 13,06278 & 9,33778 & 23,00 & 73 & 6,70 & 31,90 & 3 & 2 & 24 & 2,88 & 0,08 & 79,3 & 9 & 6 & 5,72 & 0,02 \\
\hline Dogodogo & forage & 12,90083 & 9,32222 & 35,00 & 87 & 6,70 & 31,70 & 4 & 2,5 & 8,8 & 3,84 & 0,01 & 48,8 & 5 & 0 & 2,2 & 0,72 \\
\hline Bassansani & forage & 13,05444 & 9,57861 & 38,15 & 93 & 6,70 & 31,70 & 4 & 2,5 & 11,2 & 3,84 & 0,15 & 54,9 & 4 & 0 & 8,8 & 0,47 \\
\hline Mallawa & forage & 13,03361 & 9,61139 & 23,00 & 180 & 6,76 & 31,70 & 17 & 2 & 10,4 & 1,46 & 0,48 & 43,92 & 2,2 & 23 & 12,32 & 0,26 \\
\hline Gai & forage & 13,48556 & 9,35556 & 9,80 & 1034 & 6,82 & 31,60 & 25 & 6 & 70,4 & 43,2 & 0,23 & 213,5 & 96 & 75 & 58,5 & 0,41 \\
\hline Makaoratchi & forage & 13,55833 & 9,33167 & 36 & 120 & 5,89 & 32,90 & 5 & 2,5 & 6,4 & 2,4 & 0,08 & 18,3 & 5 & 0 & 29,92 & 0,16 \\
\hline Yaouri & puit & 13,26111 & 8,56722 & 18,64 & 86 & 6,20 & 28,00 & 7 & 7 & 5,6 & 1,92 & 0,19 & 48,8 & 2 & 0 & 3,08 & 0,04 \\
\hline Gouna & forage & 13,58450 & 9,12425 & 5,60 & 155 & 6,90 & 32,00 & 7 & 3 & 12 & 3,3 & 0,01 & 39,65 & 5 & 5 & 21,12 & 0,7 \\
\hline Koaya & puit & 13,19556 & 8,64889 & 15,25 & 82 & 6,10 & 29,00 & 4 & 2,5 & 20 & 6,7 & 0,07 & 85,4 & 7 & 4 & 1,76 & 0,65 \\
\hline Sassoumbroum & puit & 13,11833 & 8,48833 & 24,53 & 46 & 5,70 & 30,50 & 3 & 0,5 & 45,6 & 2,8 & 0,07 & 158,6 & 2 & 0 & 5,72 & 0 \\
\hline Yékoua & forage & 13,04028 & 8,67861 & 17,97 & 78 & 5,80 & 30,00 & 4 & 2,5 & 44 & 3,84 & 0,03 & 152,5 & 2 & 0 & 3,52 & 0 \\
\hline Matamey & puit & 13,41917 & 8,48389 & 18,40 & 56 & 5,85 & 30,00 & 11 & 4 & 4 & 0,48 & 0,07 & 35,5 & 2 & 2 & 11,44 & 0,11 \\
\hline Tsaouni & puit & 13,33972 & 8,44528 & 30,10 & 24 & 5,70 & 29,80 & 4 & 0,7 & 4 & 0,48 & 0,11 & 12,5 & 0,5 & 1 & 12,32 & 0,12 \\
\hline Godo Haoussa & puit & 13,42028 & 8,53417 & 17,77 & 152 & 6,90 & 28,00 & 7 & 4 & 25,6 & 3,36 & 0,07 & 73,2 & 8 & 1 & 24,64 & trace \\
\hline Illala B Abdou & forage & 13,61639 & 9,43556 & 16,3 & 538 & 7,56 & 31,10 & 30 & 7,5 & 32 & 20,64 & 0,25 & 244 & 14 & 19 & 27,28 & 0,64 \\
\hline
\end{tabular}


I. SANDAO et al. / Int. J. Biol. Chem. Sci. 12(4): 1931-1954, 2018

Tableau 3 : Résultats des analyses chimiques des échantillons des eaux souterraines, prélevés au cours de la campagne d'échantillonnage d'octobre 2011.

\begin{tabular}{|c|c|c|c|c|c|c|c|c|c|c|c|c|c|c|c|c|c|c|}
\hline \multirow[b]{2}{*}{$\mathbf{N}^{\circ}$} & \multirow[b]{2}{*}{ Localité } & \multirow[b]{2}{*}{ PEM } & \multirow[b]{2}{*}{ Latiude } & \multirow[b]{2}{*}{ Longitude } & \multirow[b]{2}{*}{ NS } & \multicolumn{3}{|c|}{ Paramètres physiques } & \multicolumn{10}{|c|}{ Paramètres chimiques en mg/l, prélèvements d'octobre 2011} \\
\hline & & & & & & $\begin{array}{c}\mathrm{C} \\
(\mathrm{ms} / \mathrm{cm})\end{array}$ & $\mathbf{p H}$ & $\begin{array}{c}\mathbf{T} \\
\left({ }^{\circ} \mathbf{C}\right)\end{array}$ & $\mathbf{N a}$ & $\mathbf{K}$ & $\mathbf{C a}$ & Mg & $\mathbf{F e}$ & $\mathrm{HCO3}$ & $\mathbf{C l}$ & SO4 & NO3 & $\mathbf{F}$ \\
\hline \multicolumn{19}{|c|}{ Echantillons de la nappe phréatique des dépôts récents } \\
\hline 1 & Gogo & puit & 13,64306 & 8,92833 & 3,00 & 217,00 & 6,50 & 26 & 20 & 5 & 24,8 & 4 & 0,56 & 85,4 & 6 & 9 & 38 & 0,62 \\
\hline 2 & Wacha & puit & 13,36583 & 9,28256 & 6,11 & 317,00 & 6,70 & 27 & 25 & 3,5 & 13 & 3,3 & 0,16 & 61 & 21 & 16 & 19 & 0,42 \\
\hline 3 & Boulbaram & forage & 13,37000 & 9,18000 & 5,50 & 397,00 & 6,50 & 27 & 27 & 6 & 37,6 & 8,6 & 0,15 & 73,2 & 23 & 51 & 46 & 0,51 \\
\hline 4 & Koutchika & puit & 13,37778 & 8,98167 & 1,91 & 385,00 & 6,80 & 27 & 50 & 10 & 26,4 & 7,2 & 0,16 & 201,3 & 8 & 19 & 2,64 & 0,76 \\
\hline 5 & Tassaou & forage & 13,50417 & 8,49194 & 5,07 & 227,00 & 6,90 & 27 & 6 & 2 & 20,8 & 1,92 & 0,03 & 67 & 9,5 & 0 & 10,1 & 0,24 \\
\hline 6 & Kounkourey & puit & 13,33222 & 8,80500 & 2,85 & 2190,00 & 7,30 & 20 & 165 & 134 & 184 & 37,44 & 0,06 & 500,2 & 170 & 260 & 12 & 0,88 \\
\hline 7 & Gomba & puit & 13,29861 & 8,75000 & 5,36 & 825,00 & 6,90 & 27 & 70 & 45 & 30,4 & 15,06 & 0,04 & 109,8 & 76 & 48 & 113,5 & 0,38 \\
\hline 8 & Bakadoubou & forage & 12,96972 & 9,04944 & 20,78 & 151,50 & 5,80 & 27 & 4 & 2,5 & 9,6 & 6,2 & 0,06 & 36,6 & 9 & 18 & 1 & 0 \\
\hline 9 & Maidamoussa & forage & 13,02222 & 8,91083 & 15,00 & 62,00 & 5,52 & 27 & 3,6 & 2 & 4 & 2 & 0,05 & 12,2 & 2 & 0 & 21 & 0 \\
\hline 10 & Bandé & puit & 13,17944 & 8,88417 & 13,54 & 147,00 & 6,23 & 27 & 2,5 & 1 & 14,4 & 5,2 & 0,09 & 73,2 & 2 & 0 & 2,2 & 0 \\
\hline 11 & Beykori & puit & 13,23056 & 8,94111 & 7,04 & 231,00 & 6,21 & 27 & 9 & 15,85 & 3,2 & 10,69 & 0,06 & 37 & 10,9 & 10 & 29,48 & 0,08 \\
\hline 12 & Bangaza & forage & 13,21000 & 9,18778 & 6,00 & 58,50 & 6,20 & 27 & 2,5 & 1 & 4,8 & 2 & 0,07 & 31,72 & 2 & 0 & 0,44 & 0 \\
\hline 13 & Dirani & forage & 13,27300 & 9,40000 & 5,00 & 195,00 & 6,12 & 27 & 6 & 6 & 14,4 & 6,7 & 0,04 & 26,62 & 8 & 0 & 60 & 0 \\
\hline 14 & Maigouaba & forage & 13,16667 & 9,31556 & 3,34 & 74,00 & 6,80 & 27 & 4 & 2 & 4 & 2 & 0,09 & 24,4 & 2 & 0 & 9 & 0,22 \\
\hline 15 & Kakibaré & forage & 13,35056 & 9,39000 & 2,00 & 157,80 & 5,20 & 27 & 21 & 1,5 & 4 & 1,4 & 0,05 & 18,3 & 12 & 2 & 31 & 0,21 \\
\hline 16 & Guidimouni & forage & 13,69994 & 9,50944 & 22,00 & 86,00 & 6,40 & 27 & 4 & 4 & 5,6 & 2,4 & 0,05 & 18,3 & 3 & 3 & 18 & 0,29 \\
\hline 17 & Gamdou & forage & 13,36472 & 9,99028 & 7,3 & 240,00 & 6,70 & 27 & 9 & 3 & 17,6 & 8,2 & 0,13 & 85,4 & 5 & 6 & 12 & 0,39 \\
\hline 18 & Kousseirie & forage & 13,54306 & 9,98306 & 25 & 108,00 & 6,50 & 27 & 4 & 2 & 7,2 & 2,4 & 0,03 & 27,4 & 3 & 0 & 11,4 & 0,32 \\
\hline 19 & Gaï & forage & 13,48556 & 9,35556 & 9,80 & 357,00 & 6,50 & 27 & 8 & 17 & 30,4 & 6,7 & 0,01 & 109,8 & 10 & 6 & 28,6 & 0,52 \\
\hline 20 & Moni & forage & 13,46306 & 9,58944 & 6,10 & 103,00 & 6,40 & 27 & 6,76 & 3,5 & 0,8 & 5,82 & 0,2 & 35,4 & 0,8 & 0 & 16,72 & 0,1 \\
\hline
\end{tabular}




\section{SANDAO et al. / Int. J. Biol. Chem. Sci. 12(4): 1931-1954, 2018}

Echantillons des nappes profondes de socle, des grès argileux de Mallawa, des grès du Continental Hamadien et des grès du Continental Terminal

\begin{tabular}{|c|c|c|c|c|c|c|c|c|c|c|c|c|c|c|c|c|c|c|}
\hline 1 & Illala Abdou & forage & 13,61639 & 9,43556 & 16,30 & 590,00 & 6,90 & 27 & 30 & 8 & 48 & 17 & 0,08 & 250,1 & 14 & 29 & 18,5 & 0,71 \\
\hline 2 & Doungas & forage & 13,06278 & 9,33778 & 23,00 & 85,00 & 6,60 & 27 & 3 & 1,5 & 8 & 2,4 & 0,03 & 29,2 & 2 & 7 & 6 & 0,33 \\
\hline 3 & Yaouri & forage & 13,26111 & 8,56722 & 14,00 & 52,00 & 6,10 & 27 & 3,6 & 1,7 & 1,2 & 0,96 & 0,05 & 7,93 & 3 & 0 & 7 & 0,22 \\
\hline 4 & DogoDogo & forage & 12,90083 & 9,32222 & 35,00 & 102,00 & 6,50 & 27 & 5 & 2,5 & 15 & 9,72 & 0,07 & 46 & 2,2 & 0 & 11 & 0,24 \\
\hline 5 & FaganKaoua & forage & 12,94500 & 9,66600 & 38,15 & 385,00 & 6,50 & 27 & 18 & 4 & 29 & 6,7 & 0,08 & 109,8 & 20 & 17 & 28 & 0,56 \\
\hline 6 & Mallawa & forage & 13,03361 & 9,61139 & 23,00 & 282,00 & 6,50 & 27 & 25 & 3,5 & 21 & 4,3 & 0,03 & 97,6 & 9 & 34 & 5 & 0,8 \\
\hline 7 & MalamGanari & forage & 13,17917 & 9,90528 & 17,60 & 496,00 & 6,60 & 27 & 50 & 4 & 43,2 & 8 & 0,04 & 183 & 5 & 79 & 4,4 & 0,64 \\
\hline 8 & Guidiguir & forage & 13,67139 & 9,83056 & 9,1 & 135,00 & 6,70 & 27 & 6,76 & 4,25 & 2,4 & 9,24 & 0,01 & 61 & 0,6 & 8 & 5,72 & 0,1 \\
\hline 10 & Baraoua B & puit & 13,59876 & 8,54004 & 38,24 & 347,00 & 6,60 & 21 & 4,13 & 11,75 & 16,8 & 7,28 & 0,53 & 99,43 & 1,1 & 6 & 8,36 & 0,13 \\
\hline 11 & Kanthé pc & puit & 13,53917 & 8,45889 & 14,22 & 265,00 & 6,80 & 27 & 9 & 10,2 & 24 & 2,88 & 0,07 & 112 & 5 & 0 & 5,28 & 0,37 \\
\hline 12 & Kantché forage & forage & 13,56000 & 8,47000 & 16,00 & 457,00 & 7,02 & 27 & 18 & 18,75 & 33,6 & 12,4 & 0,03 & 207,4 & 3,4 & 14 & 6,16 & 0,67 \\
\hline 13 & Tsaouni & forage & 13,33972 & 8,44528 & 30,10 & 51,00 & 5,70 & 21 & 2 & 0,5 & 3,2 & 0,96 & 0,06 & 12,2 & 3 & 0 & 4 & 0,29 \\
\hline 14 & $\begin{array}{l}\text { Godo Haooussa } \\
\mathrm{f}\end{array}$ & forage & 13,42028 & 8,53417 & 21,00 & 158,00 & 5,60 & 26 & 5 & 1,5 & 8,8 & 2,88 & 0,05 & 22,5 & 7,5 & 0 & 12 & 0,28 \\
\hline 15 & $\begin{array}{l}\text { Godo Haussa } \\
\text { pc }\end{array}$ & puit & 13,42028 & 8,53517 & 21,15 & 192,00 & 6,00 & 20 & 7 & 3 & 12,8 & 2,88 & 0,04 & 36,6 & 7 & 0 & 14 & 1,3 \\
\hline 16 & Koaya & puit & 13,19556 & 8,64889 & 14,84 & 91,00 & 5,40 & 27 & 4 & 2,5 & 6,2 & 1 & 0,19 & 24,4 & 6,5 & 4 & 0,44 & 0,04 \\
\hline 17 & Sbroum & puit & 13,11833 & 8,48833 & 23,65 & 54,00 & 5,29 & 27 & 2,5 & 1 & 2,4 & 1,2 & 0,08 & 15,86 & 2 & 0 & 0,44 & 0,03 \\
\hline 18 & Yékoua & forage & 13,04028 & 8,67861 & 17,97 & 85,00 & 5,42 & 27 & 2,5 & 2 & 4 & 2,88 & 0,06 & 26,84 & 2 & 0 & 4,4 & 0 \\
\hline 19 & Dan Tchiaou & forage & 12,88028 & 9,09278 & 21,45 & 54,00 & 5,72 & 27 & 2 & 1,5 & 3,2 & 2,88 & 0,04 & 24,4 & 2 & 0 & 2,64 & 0,03 \\
\hline 20 & Baboujé & forage & 13,26111 & 9,61944 & 13,00 & 192,80 & 6,50 & 27 & 25 & 3 & 14,4 & 2,4 & 0,38 & 97,6 & 3 & 6 & 9 & 0,32 \\
\hline 21 & Gouchi & forage & 13,51472 & 9,54972 & 15,00 & 191,00 & 6,50 & 27 & 5 & 4 & 17,6 & 7,6 & 0,03 & 85,4 & 4 & 0 & 14 & 0,33 \\
\hline 22 & Gouna & forage & 13,58450 & 9,12425 & 5,60 & 180,00 & 6,14 & 26 & 12 & 2,5 & 12 & 3,3 & 0 & 36,6 & 3 & 5 & 35 & 0,55 \\
\hline
\end{tabular}




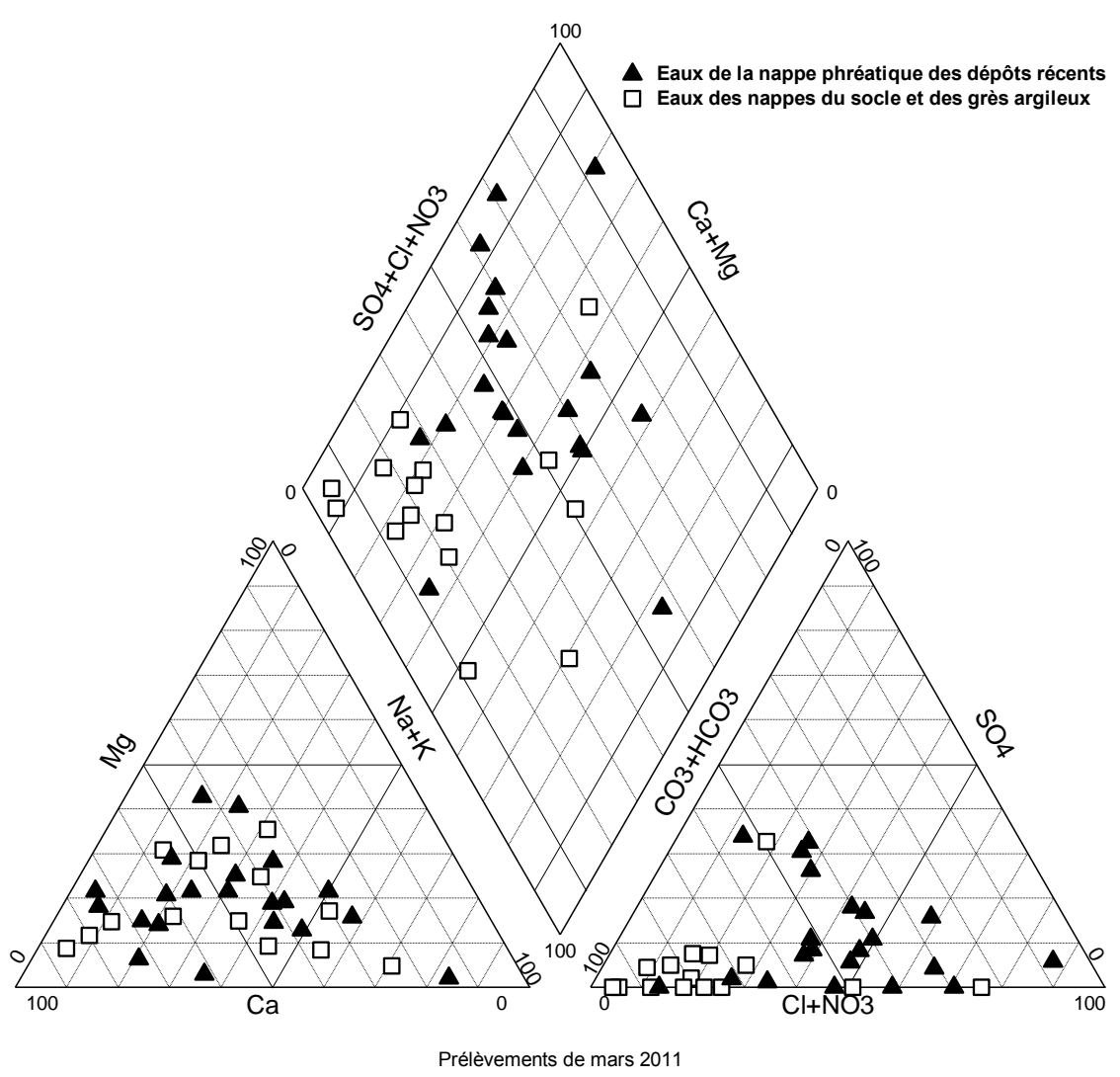

Figure 5 : Diagramme de Piper des eaux, pour les analyses de mars 2011.

Tableau 4 : Teneurs en isotopes ${ }^{18} \mathrm{O}$ et ${ }^{2} \mathrm{H}$ des eaux souterraines : campagne de mars 2011.

\begin{tabular}{llllllllll}
\hline \multicolumn{1}{l}{ Campagne 1 mars 2011: eaux de la nappe phréatique des Alluvions récentes de la Korama } \\
\hline $\mathbf{N}^{\circ}$ & Localité & Ouvrage & Latitude & Longitude & $\mathbf{N S}$ & $\mathbf{C l}$ & $\boldsymbol{\delta}^{\mathbf{1 8}} \mathbf{O}$ & $\boldsymbol{\delta}^{\mathbf{2}} \mathbf{H}$ & $\mathbf{d}$ \\
\hline 1 & Kounkourey & puit & 13,3322 & 8,8050 & 3,24 & 4,00 & $-3,35$ & $-23,38$ & 3 \\
2 & Koutchika & puit & 13,3778 & 8,9817 & 1,05 & 0,35 & $-1,98$ & $-18,73$ & -3 \\
3 & Gogo & puit & 13,6431 & 8,9283 & 3,00 & 0,20 & $-4,98$ & $-30,58$ & 9 \\
4 & Bandé puit & puit & 13,1794 & 8,8842 & 13,73 & 0,11 & $-4,50$ & $-31,18$ & 5 \\
5 & Beykori pc & puit & 13,2306 & 8,9411 & 7,18 & 0,39 & $-4,18$ & $-27,75$ & 6 \\
6 & Maigowaba & puit & 13,1667 & 9,3156 & 3,34 & 0,20 & $-3,92$ & $-27,46$ & 4 \\
7 & Wachapuit & puit & 13,3658 & 9,2826 & 10,00 & 1,41 & $-4,09$ & $-30,22$ & 3 \\
8 & Tassaou & forage & 13,5042 & 8,4919 & 5,00 & 0,28 & $-3,83$ & $-26,12$ & 5 \\
9 & Doungou & forage & 13,4108 & 8,6378 & 1,70 & 0,05 & $-4,15$ & $-27,17$ & 6 \\
10 & Maïdamoussa & forage & 13,0222 & 8,9108 & 15,00 & 0,11 & $-4,60$ & $-30,24$ & 7 \\
12 & Bangaza & forage & 13,2100 & 9,1878 & 6,00 & 0,06 & $-4,48$ & $-32,14$ & 4 \\
13 & Kakibaré & forage & 13,3506 & 9,3900 & 2,00 & 0,17 & $-4,21$ & $-28,47$ & 5 \\
14 & Boulbaram & forage & 13,3700 & 9,1800 & 5,50 & 0,31 & $-3,13$ & $-22,15$ & 3 \\
15 & Guidimouni AEP & forage & 13,6999 & 9,5094 & 22,00 & 0,08 & $-3,34$ & $-23,20$ & 3 \\
16 & Koaya & puit & 13,1956 & 8,6489 & 15,25 & 0,20 & $-4,06$ & $-28,32$ & 4
\end{tabular}




$\begin{array}{llllllllll}17 & \text { Godo haoussa } & \text { puit } & 13,4203 & 8,5342 & 17,77 & 0,23 & -3,92 & -27,30 & 4 \\ 18 & \text { Yaouri } & \text { puit } & 13,2611 & 8,5672 & 18,64 & 0,06 & -3,65 & -26,81 & 2 \\ 19 & \text { Gai } & \text { forage } & 13,4856 & 9,3556 & 9,80 & 2,71 & -4,23 & -30,46 & 3\end{array}$

Campagne 1, mars 2011: eaux des nappes du socle et des grès argileux du Continental et de Mallawa

\begin{tabular}{llllllllll}
$\mathbf{N}^{\circ}$ & Localité & Ouvrage & Latitude & Longitude & $\mathbf{N S}$ & $\mathbf{C l}$ & $\boldsymbol{\delta}^{\mathbf{1 8}} \mathbf{O}$ & $\boldsymbol{\delta}^{\mathbf{2}} \mathbf{H}$ & $\mathbf{d}$ \\
1 & Kantché pc & puit & 13,5392 & 8,4589 & 13,96 & 0,21 & $-4,54$ & $-33,44$ & 3 \\
2 & Baraoua Bougagé & forage & 13,5988 & 8,5400 & 40,00 & 0,11 & $-5,95$ & $-42,05$ & 6 \\
3 & Gouna & forage & 13,5845 & 9,1243 & 5,60 & 0,14 & $-3,48$ & $-23,92$ & 4 \\
4 & Yékoua & forage & 13,0403 & 8,6786 & 17,97 & 0,06 & $-4,73$ & $-30,39$ & 7 \\
5 & Dan tchiao & forage & 12,8803 & 9,0928 & 21,45 & 0,08 & $-4,58$ & $-29,88$ & 7 \\
6 & Doungas & forage & 13,0628 & 9,3378 & 23,00 & 0,25 & $-4,60$ & $-32,06$ & 5 \\
7 & Dogodogo & forage & 12,9008 & 9,3222 & 35,00 & 0,14 & $-5,05$ & $-34,37$ & 6 \\
8 & Bassansani & forage & 13,0544 & 9,5786 & 38,15 & 0,11 & $-3,84$ & $-26,24$ & 4 \\
9 & Makaoratchi & forage & 13,5583 & 9,3317 & 36,00 & 0,14 & $-4,96$ & $-32,63$ & 7 \\
10 & Mallawa & forage & 13,0336 & 9,6114 & 23,00 & 0,06 & $-3,40$ & $-23,16$ & 4 \\
11 & Illala B Abdou & forage & 13,6164 & 9,4356 & 16,30 & 0,39 & $-3,76$ & $-26,76$ & 3 \\
\hline
\end{tabular}

Tableau 5 : Teneurs en isotopes ${ }^{18} \mathrm{O}$ et ${ }^{2} \mathrm{H}$ des eaux souterraines : campagne d'octobre 2011.

\begin{tabular}{llllllllll}
\hline \multicolumn{2}{l}{ Campagne 2, octobre 2011: eaux de la nappe phréatique des Alluvions récentes de la Korama } \\
\hline $\mathbf{N}^{\circ}$ & Localité & Ouvrage & Latiude & Longitude & NS & Cl & $\boldsymbol{\delta}^{18} \mathbf{O}$ & $\boldsymbol{\delta}^{\mathbf{2}} \mathbf{H}$ & $\mathbf{d}$ \\
\hline 1 & Kounkourey & puit & 13,3322 & 8,8050 & 2,85 & 4,79 & $-3,44$ & $-26,00$ & 2 \\
2 & Gomba & puit & 13,2986 & 8,7500 & 5,36 & 2,14 & $-3,80$ & $-26,12$ & 4 \\
3 & Bandé & puit & 13,1794 & 8,8842 & 13,54 & 0,06 & $-4,17$ & $-28,01$ & 5 \\
4 & Beykori & puit & 13,2306 & 8,9411 & 7,04 & 0,31 & $-4,10$ & $-27,00$ & 6 \\
5 & Wacha & puit & 13,3658 & 9,2826 & 6,11 & 0,59 & $-3,90$ & $-26,58$ & 5 \\
6 & Koutchika & puit & 13,3778 & 8,9817 & 1,91 & 0,23 & $-1,65$ & $-16,54$ & -3 \\
7 & Gogo & puit & 13,6431 & 8,9283 & 3,00 & 0,17 & $-2,32$ & $-12,20$ & 6 \\
8 & Tassaou & forage & 13,5042 & 8,4919 & 5,07 & 0,27 & $-3,88$ & $-27,78$ & 3 \\
9 & Baka doubou & forage & 12,9697 & 9,0494 & 20,78 & 0,25 & $-4,30$ & $-29,57$ & 5 \\
10 & Maidamoussa & forage & 13,0222 & 8,9108 & 15,00 & 0,06 & $-4,48$ & $-30,41$ & 5 \\
11 & Bangaza & forage & 13,2100 & 9,1878 & 6,00 & 0,06 & $-3,70$ & $-29,34$ & 0 \\
12 & Dirani & forage & 13,2730 & 9,4000 & 5,00 & 0,23 & $-3,47$ & $-22,00$ & 6 \\
13 & Kakibaré & forage & 13,3506 & 9,3900 & 2,00 & 0,34 & $-4,17$ & $-29,67$ & 4 \\
14 & Guidimouni & forage & 13,6999 & 9,5094 & 22,00 & 0,08 & $-3,29$ & $-24,00$ & 2 \\
15 & Maigouaba & forage & 13,1667 & 9,3156 & 3,34 & 0,06 & $-3,92$ & $-27,03$ & 4 \\
16 & Boulbaram & forage & 13,3700 & 9,1800 & 5,50 & 0,65 & $-3,81$ & $-27,16$ & 3 \\
17 & Godo haooussa pc & puit & 13,4203 & 8,5352 & 21,15 & 0,197 & $-3,88$ & $-27,22$ & 4 \\
18 & Koaya & puit & 13,1956 & 8,6489 & 14,84 & 0,183 & $-4,28$ & $-27,52$ & 7 \\
19 & Moni & forage & 13,4631 & 9,5894 & 6,10 & 0,02 & $-4,49$ & $-30,10$ & 6 \\
20 & Gouchi & forage & 13,5147 & 9,5497 & 15,00 & 0,113 & $-4,13$ & $-29,55$ & 3 \\
21 & Yaouri & forage & 13,2611 & 8,5672 & 14,00 & 0,085 & $-3,60$ & $-27,80$ & 1 \\
22 & Guidiguir & forage & 13,6714 & 9,8306 & 9,10 & 0,017 & $-4,03$ & $-29,77$ & 2 \\
23 & Gamdou & forage & 13,3647 & 9,9903 & 7,30 & 0,14 & $-4,11$ & $-29,39$ & 4 \\
24 & Kousseirie & forage & 13,5431 & 9,9831 & 25,00 & 0,08 & $-4,44$ & $-30,16$ & 5 \\
& & & & 1944 & & & & &
\end{tabular}




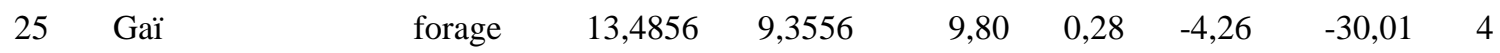

Campagne 2, octobre 2011: eaux des nappes du socle et des grès argileux du Continental et de Mallawa

\begin{tabular}{|c|c|c|c|c|c|c|c|c|c|}
\hline $\mathbf{N}^{\circ}$ & Localité & Ouvrage & Latiude & Longitude & NS & Cl & $\delta^{18} \mathrm{O}$ & $\delta^{2} \mathbf{H}$ & d \\
\hline \multirow[t]{2}{*}{1} & Baraoua & puit & 13,5988 & 8,5400 & 38,24 & 0,031 & $-5,87$ & $-41,46$ & \\
\hline & bougagé & & & & & & & & 5 \\
\hline 2 & Kantché pc & puit & 13,5392 & 8,4589 & 14,22 & 0,141 & $-4,62$ & $-32,13$ & 5 \\
\hline 3 & Gouna & forage & 13,5845 & 9,1243 & 5,60 & 0,085 & $-3,38$ & $-25,87$ & 1 \\
\hline 4 & Sbroum & puit & 13,1183 & 8,4883 & 23,65 & 0,056 & $-4,90$ & $-33,90$ & 5 \\
\hline 5 & Kantché forage & forage & 13,5600 & 8,4700 & 16,00 & 0,096 & $--4,82$ & $-32,23$ & 6 \\
\hline 6 & Tsaouni & forage & 13,3397 & 8,4453 & 30,10 & 0,085 & $-4,86$ & $-31,36$ & 8 \\
\hline \multirow[t]{2}{*}{7} & Godo haoussa & forage & 13,4203 & 8,5342 & 21,00 & 0,212 & $-4,66$ & $-29,07$ & \\
\hline & forage & & & & & & & & 8 \\
\hline 8 & Malam Ganari & forage & 13,1792 & 9,9053 & 17,60 & 0,14 & $-4,44$ & $-28,27$ & 7 \\
\hline 9 & Yékoua & forage & 13,0403 & 8,6786 & 17,97 & 0,056 & $-4,53$ & $-30,41$ & 6 \\
\hline 10 & Baboujé & forage & 13,2611 & 9,6194 & 13,00 & 0,08 & $-4,42$ & $-31,93$ & 3 \\
\hline 11 & Makaouratchi & forage & 13,5583 & 9,3317 & 36,00 & 0,34 & $-5,11$ & $-36,00$ & 5 \\
\hline 12 & Dan Tchiaou & forage & 12,8803 & 9,0928 & 21,45 & 0,056 & $-4,56$ & $-31,62$ & 5 \\
\hline 13 & Illala Abdou & forage & 13,6164 & 9,4356 & 16,30 & 0,395 & $-3,63$ & $-26,07$ & 3 \\
\hline 14 & Doungas & forage & 13,0628 & 9,3378 & 23,00 & 0,056 & $-4,53$ & $-30,50$ & 6 \\
\hline 15 & Dogo Dogo & forage & 12,9008 & 9,3222 & 35,00 & 0,062 & $-4,91$ & $-35,53$ & 4 \\
\hline 16 & Fagan Kaoua & forage & 12,9450 & 9,6660 & 38,15 & 0,564 & $-4,87$ & $-35,93$ & 3 \\
\hline 17 & Mallawa & forage & 13,0336 & 9,6114 & 23,00 & 0,254 & $-5,36$ & $-38,36$ & 5 \\
\hline
\end{tabular}

Tableau 6: Teneurs en Tritium ${ }^{3} \mathrm{H}$ et ${ }^{13} \mathrm{Cet}$ activités en ${ }^{14} \mathrm{C}$ des eaux souterraines, mars et octobre 2011.

\begin{tabular}{|c|c|c|c|c|}
\hline Nom du site & Nappe captée & $\begin{array}{c}\text { Teneur en }{ }^{3} \mathbf{H} \\
\text { (UT) }\end{array}$ & Activités ${ }^{14} \mathrm{C}(\%)$ & $\begin{array}{l}\text { Teneur en } \\
{ }^{13} \mathrm{C}(\delta \%)\end{array}$ \\
\hline & & \multicolumn{3}{|c|}{ Prélèvements de mars 2011} \\
\hline Guidimouni AEP & $\begin{array}{l}\text { Phréatique des } \\
\text { alluvions }\end{array}$ & $5 \pm 0,5$ & & \\
\hline Barawa Bougagé PC & Continental Hamadien & $<0,6$ (trace) & & \\
\hline Makaouratchi Forage & Socle quartzitique & $<0,6$ (trace) & & \\
\hline \multirow[t]{2}{*}{ Mallawa Forage AEP } & Grès de Mallawa & $0,8 \pm 0,4$ & & \\
\hline & & \multicolumn{3}{|c|}{ Prélèvements d'octobre 2011} \\
\hline Kantché forage & Continental Hamadien & & $101,9 \pm 0,4$ & $-10,15$ \\
\hline Mallawa Forage AEP & Grès de Mallawa & & $37,1 \pm 0,3$ & $-8,88$ \\
\hline Gouna Forage AEP & Socle granitique & & $98,4 \pm 0,4$ & $-11,66$ \\
\hline Makaouratchi Forage & Socle quartzitique & & $84,2 \pm 03$ & $-12,9$ \\
\hline
\end{tabular}




\section{DISCUSSION}

\section{Comportement hydrogéochimique}

Les eaux des aquifères étudiés montrent des faciès diversifiés (Figure 5). Cette diversité des faciès est liée surtout aux types des matériaux aquifères, mais aussi à l'hydrodynamique des nappes. La signature hydrogéochimique de ces eaux peut être utilisée pour expliquer cette dynamique. Le faciès chloruro-sulfaté calcique voire nitraté des eaux est majoritairement observé au niveau des nappes phréatiques. L'évolution de ces faciès vers le pôle chloruré-nitraté est principalement due à une augmentation des teneurs en nitrates. Or, les eaux chloruréesnitratées sont interprétées comme caractéristiques d'une nappe à recharge récente (Abdou Babaye et al., 2018). Par contre, l'évolution des eaux de la zone bicarbonatée calco-magnésienne vers le domaine bicarbonaté sodique pourrait être un signe de vieillissement des eaux dû aux indices d'échange de base entre les alcalinoterreux et les alcalins. Le contact prolongé de l'eau chargée en $\mathrm{CO}_{2}$ avec l'encaissant se traduit par un enrichissement en cations majeurs $\left(\mathrm{Ca}^{2+}, \mathrm{Mg}^{2+}, \mathrm{Na}^{+}, \mathrm{K}^{+}\right)$et en ions bicarbonates (Abdou Babaye et al., 2016, 2018).

Les diagrammes $\mathrm{pH}$ vs $\mathrm{Fe}$ avec les valeurs de la campagne d'avril 2017 (Figure 6) montrent que le $\mathrm{pH}$ des eaux serait contrôlé par les valeurs des ions Fe. Les eaux les plus acides se retrouvent dans les secteurs d'abondance des ions Fe. Ainsi, les valeurs basses du $\mathrm{pH}$ dans les nappes profondes des grès sidérolitiques s'expliqueraient par les phénomènes de précipitation de la sidérite $\left(\mathrm{FeCO}_{3}\right)$ et des ions $\mathrm{H}^{+}$dans les niveaux ferrugineux au contact de l'eau, hypothèse déjà émise par Zakara (1990). Les valeurs les plus élevées des $\mathrm{pH}$, dans les nappes phréatiques de profondeur moyenne, contenues dans les grès de Mallawa et dans celles du socle quartzique seraient des indices de faibles interactions avec la lithologie.

Les valeurs médianes des $\mathrm{pH}$ rencontrées dans la nappe des alluvions récentes seraient issues des mélanges avec des eaux de précipitations atmosphériques après leur infiltration.

La Figure 7 montre que la conductivité varie en fonction des saisons. L'amplitude des fluctuations de la conductivité au cours de la saison pluvieuse, peut atteindre $500 \mu \mathrm{S} / \mathrm{cm}$ au niveau des sites de Gomba Haoussa et Kounkourey, au centre du bassin, dans la nappe des alluvions récentes. Cela peut s'expliquer par le lessivage des niveaux non saturés au cours des infiltrations des eaux des pluies efficaces. Il est à rappeler qu'en contexte sahélien, l'évaporation accélérée des précipitations de faible intensité engendre l'accumulation de résidus chimiques dans la zone non saturée. Ces éléments seront remis en solution et charriés vers les nappes par des précipitations lorsqu'elles sont importantes (Abdou Babye, 2012). Ces éléments chimiques arrivent dans les nappes selon la loi de tous ou rien (Sandao, 2013), avec comme effet, l'augmentation brusque de la charge minérale et donc de la conductivité.

Dans les autres aquifères (puits de Barawa Bougagé indiqué sur la Figure 6), les amplitudes de variation de la conductivité sont très faibles, et pourraient s'expliquer par une faible contribution des précipitations actuelles dans le renouvellement des eaux de ces nappes.

Dans la nappe phréatique des dépôts récents, les cations $\mathrm{Ca}^{++}$et $\mathrm{Mg}^{++}$sont toujours dominants, mais les anions $\mathrm{SO}_{4}^{--}, \mathrm{Cl}^{-}$et $\mathrm{NO}_{3}^{-}$ ont tendance à se substituer aux anions $\mathrm{HCO}_{3}{ }^{--}$. Le faciès tend vers le pôle nitrateschlorures-sulfates, avec des $\mathrm{pH}$ acides portant le cachet des infiltrations des eaux atmosphériques. La minéralisation serait ici, plus contrôlée par le processus de concentration des éléments chimiques contenus dans les précipitations, par évaporation avant et au cours de leurs infiltration (Guéro, 2003). Les proportions importantes en nitrates et en sodium peuvent s'expliquer en partie par la pollution anthropique de la nappe, à cause des faibles profondeurs du niveau statique et d'une lithologie sableuse (Abdou Babaye et al., 2016). Cela renforce l'hypothèse de la 
recharge de cette nappe par l'infiltration des eaux atmosphériques.

Les valeurs de la minéralisation, particulièrement élevées au niveau de trois (3) ouvrages (piézomètre de Kounkourey, puits cimenté de Gomba Haoussa et forage de Gaï), résulteraient surtout des impacts des environs immédiats des ouvrages et de leur utilisation pour l'abreuvement du bétail. En effet, la forte anthropisation et les conditions d'exploitation de ces ouvrages favorisent la décomposition de la matière organique et l'infiltration des éléments chimiques et biologiques dans la nappe (Abdou Babaye et al., 2016). Cette observation illustre bien l'apport des ions chimiques par les eaux d'infiltration (Hamza et al., 2007 ; Lasm et al., 2011).

\section{Processus de recharge}

Dans les régions arides, la composition isotopique des eaux souterraines peut être considérablement modifiée par le phénomène d'évaporation par rapport à celles des précipitations locales (Clark et Fritz, 1997 ; Favreau et al., 2002). Mais, malgré le taux d'ETP élevées, il est possible d'avoir des eaux souterraines ayant des compositions isotopiques proches de la composition moyenne des précipitations, démontrant des flux de recharge rapides et contemporaines vers les aquifères (Acheamponga et Hess, 2000 ; Goni, 2006).

La Figure 8 présente les diagrammes $\delta^{2} \mathrm{H}$ vs $\delta^{18} \mathrm{O}$ des eaux de la zone d'étude en mars et octobre 2011. Ils montrent que les points représentant les eaux souterraines se placent en dessous de la droite locale des eaux des précipitations (DML), esquissée avec les précipitations de 2011 à la station de Zinder, et qui est elle-même en dessous de la droite météorique mondiale (DMM). Ce qui prouve que les masses d'air océanique à l'origine des précipitations dans la zone, ont subi des processus d'évaporation au cours de leur trajet vers le continent (Favreau et al., 2002; Tremoy et al., 2012).

Les eaux de la nappe des alluvions récentes définissent un ensemble assez homogène, hormis deux (2) points qui se singularisent (le puits cimentés de Koutchika et celui de la vallée de Gogo). Les points représentant les échantillons des eaux de cette nappe se regroupent en dessous de la DML pour définir deux (2) droites d'évaporation de pente 4,76 et 6,43 respectivement en octobre et mars 2011. Les positions de ces droites indiqueraient que ces eaux portent des cachets d'évaporation ayant affecté les précipitations qui sont à leurs origines. Edmunds et al. (2002), Stadler et al. (2010) et Abdou Babaye et al. (2018) ont également trouvé des pentes proches de ces valeurs pour la droite d'évaporation. L'augmentation de la valeur de la pente pourrait être liée à l'importance progressive des apports des précipitations entre octobre et mars, ce qui confirmerait l'hypothèse de la minéralisation des eaux de cette nappe des sables alluvionnaires par concentration et infiltration des éléments chimiques atmosphériques.

Le puits cimenté de Koutchika est situé dans le lit de la grande rivière Korama. Pour les deux campagnes, les points correspondant se placent loin des autres sur la droite météorique locale, avec des valeurs plus élevées en isotopes lourds, proches de celles des eaux de surface de la zone (Sandao, 2013). Ce qui pourrait être expliqué par l'importance des apports des eaux de surface dans la recharge de cette nappe phréatique dans ce secteur (Goni, 2006 ; Abdou Babaye, 2012).

Le puits cimenté de la vallée de Gogo est situé dans un secteur où la nappe est soumise à des pompages intensifs de façon continue avec des débits de plus de 10000 $\mathrm{m}^{3} /$ jour pour l'alimentation de la ville de Zinder. Les fluctuations piézométriques saisonnières dans ce secteur peuvent atteindre le mètre, selon l'importance de la pluviométrie. Pour les prélèvements de mars 2011, l'eau de ce puits qui capte la nappe alluvionnaire, se situe sur la DML et présente des caractéristiques isotopiques des nappes plus profondes (des grès du Continental Hamadien), avec des teneurs relativement faibles en ${ }^{18} \mathrm{O}$ et ${ }^{2} \mathrm{H}$. Par contre, l'échantillon prélevé en octobre 2011, a des teneurs élevées en ${ }^{18} \mathrm{O}$ et ${ }^{2} \mathrm{H}$, et se place toujours sur la DML, indiquant pour cette période, qu'il s'agit d'une 
eau avec apport des percolations rapides des pluies locales. Tout laisse supposer que la dépression piézométrique de la nappe phréatique résultant des pompages intensifs et la densité du réseau de fractures du socle dans cette partie du bassin recouvert de sable filtrant, permettent des écoulements importants (par drainance ascendante) des eaux de la nappe des grès du Continental Hamadien, pauvres en ${ }^{18} \mathrm{O}$ et ${ }^{2} \mathrm{H}$ vers la nappe phréatique des alluvions récentes, dont les eaux sont enrichies en isotopes lourds. Ce qui corrobore l'hypothèse d'interconnections hydrauliques locales entre les nappes, ceci à travers les fractures du socle et les grès argileux du Continental Hamadien (Sogetha, 1964 ; Zakara, 1990).

Les eaux des nappes des grès du Continental Hamadien et des grès de Mallawa se placent en dessous des droites météoriques locales ( DML), avec des droites d'évaporation des eaux dont les pentes sont de 6,88 en mars et 7,52 en octobre et des valeurs moyennes des excès en deutérium de $5 \%$ pour les deux campagnes de prélèvement. Cela reflète aussi un cachet d'évaporation des précipitations ayant affecté ces eaux, mais le processus serait ici plus complexe. Il peut être expliqué par une infiltration très lente et pendant des périodes longues des eaux évaporées à la surface, ceci à travers la nappe phréatique susjacente (Edmunds et al., 2002 ; Stadler et al., 2010).

L'augmentation de la pente de la droite des eaux après la saison des pluies, peut s'expliquer par des phénomènes d'écoulements souterrains et de mélanges avec des eaux plus enrichies de la nappe phréatique, le sens des écoulements entre les nappes des grès de Mallawa et du Continental Hamadien et la nappe phréatique étant toujours fonction de la dynamique et de la piézométrie de la nappe superficielle. Les variations des teneurs isotopiques sont ainsi lissées dans le temps au niveau des nappes des grès, plus profonde.

Cependant, les seuls processus d'évaporation des précipitations dans un climat comme l'actuel, ne peuvent pas expliquer la position de ces eaux dans les diagrammes $\delta^{2} \mathrm{H}$ vs $\delta^{18} \mathrm{O}$, compte tenu de leurs faibles teneurs en isotopes lourds, inférieures à celles des pluies contemporaines.

En effet, les valeurs moyennes des excès en deutérium de $5 \%$ dans les nappes plus profondes du bassin de la Korama sont dans les mêmes ordres de grandeur que celles trouvées dans les nappes profondes de la bande sahélienne, 7,5\%, d'après Guéro (2003) et Goni (2006). Elles correspondraient à des eaux des précipitations ayant intervenu au cours d'un paléoclimat humide, mais assez chaud, l'humidité relative de l'air en cette période serait de plus de $5 \%$ plus élevée que celle du climat actuel.

Les résultats des reconstitutions paléoclimatiques (Guéro, 2003) s'accordent à dire que pour trouver un tel climat humide et moins froid au Sahel, il faut remonter entre 18000 et 10000 ans BP.

Aussi, la reconstitution faite pour la région sahélienne par Stéphanie (2000), à partir des teneurs en isotopes lourds des eaux des nappes et des températures moyennes de leur recharge (déduites des teneurs en gaz rares) a montré que les eaux ayant des valeurs proches en $\delta^{18} \mathrm{O}(-5 \%), \delta^{2} \mathrm{H}(-32 \%)$, et des excès en deutérium $(5 \%)$ obtenues dans les nappes plus profondes de la zone d'étude, sont datées entre 18000 et 10000 ans BP. En tenant compte des incertitudes déjà évoquées et des effets des mélanges avec des eaux de la nappe phréatique plus enrichies en isotopes lourds, on peut envisager le même paléoclimat, de 12000 à 10000 ans BP.

Les eaux de la nappe phréatique sont représentées par des points qui définissent des droites des eaux dont les pentes sont de 4,99 en mars (période des basses eaux) et 5,96 en octobre (en hautes eaux) (Figure 6), indiquant les effets d'une plus forte évaporation. Ces points constituent un ensemble assez homogène, hormis les deux (2) points cités plus haut qui se singularisent de nouveau (puits cimenté de Koutchika et celui de la vallée de Gogo). Les points de la nappe phréatique sont proches de la droite locale des précipitations, et leur aire de répartition s'aplatit en mars, pendant la période des basses eaux. Ils se situeraient dans les aires de 
recharge de la nappe par infiltration directe des eaux des pluies plus ou moins évaporées. $\mathrm{Au}$ cours de l'étiage, et surtout dans les secteurs des prélèvements intensifs par pompage, on assisterait à des apports par drainance ascendante des eaux des nappes plus profondes et appauvries en isotopes lourds.

\section{Essai de datation des eaux souterraines de la zone d'étude. \\ Analyse des teneurs en tritium}

Les teneurs en tritium sont corrélables avec celles trouvées récemment dans d'autres zones du Niger, notamment dans les nappes libres des alluvions de Dosso, avec une moyenne de 6 UT en 1997 et des valeurs inférieures au seuil détectable dans les eaux du Continental Hamadien (Guéro, 2003).

En considérant que la valeur de 5 UT est caractéristique des eaux des précipitations actuelles dans la sous-région (GNIP, 2011), les teneurs en ${ }^{3} \mathrm{H}$ trouvées dans les échantillons permettent de classer les eaux souterraines de la zone d'étude en deux groupes :

- les eaux contenant du tritium autour de 5 UT, correspondant à l'échantillon du forage de Guidimouni, captant la nappe phréatique des alluvions récentes. Ces eaux peuvent être considérées essentiellement à composante récente, issue de l'infiltration des précipitations locales ;

- les eaux sans tritium avec des valeurs autour de zéro, ce sont les eaux des forages de Barawa Bougaé (dans la nappe du Continental Hamadien), de Mallawa (dans la nappe des grès de Mallawa) et de Makaouratchi (dans une nappe des fractures $\mathrm{du}$ socle). Pour les nappes profondes des grès, elles se seraient essentiellement rechargées à partir des précipitations d'avant le pic du tritium de 1963. Pour les eux de la nappe du socle, il pourrait être les effets de la drainance ascendante.

\section{Activités et les teneurs en isotopes du carbone ${ }^{14} \mathrm{C}$ et ${ }^{13} \mathrm{C}$}

Le radiocarbone a également une origine atmosphérique et une origine artificielle liée aux activités nucléaires. Dans le bassin de la Korama, il n'existe aucune autre source artificielle depuis les essais nucléaires des années 1960. Ainsi, après correction pour tenir compte des processus biologiques et minéralogiques dans les aquifères, les activités en ${ }^{14} \mathrm{C}$ élevées (proches ou supérieures à 100\%) dans les eaux souterraines sont des indices d'une rémanence du pic nucléaire des années 1962-1964, donc des eaux essentiellement contemporaines. Les faibles valeurs de l'activité ${ }^{14} \mathrm{C}$ pourraient caractériser des eaux d'avant le pic.

Les teneurs en ${ }^{13} \mathrm{C}$ permettent d'effectuer les corrections nécessaires et de discuter de la part du CITD atmosphérique dans les activités du ${ }^{14} \mathrm{C}$.

Ainsi, à la suite des premières interprétations des teneurs en ${ }^{18} \mathrm{O},{ }^{2} \mathrm{H}$ et ${ }^{3} \mathrm{H}$ pour les échantillons de la première campagne, il a été jugé nécessaire de prévoir pour la seconde campagne, l'analyse des isotopes du carbone dans les nappes plus profondes. Pour cela, quatre (4) échantillons ont été prélevés en octobre 2011, dans les nappes dont les eaux sont dépourvues du tritium. Les résultats des analyses $\mathrm{du}{ }^{14} \mathrm{C}$ et ${ }^{13} \mathrm{C}$ sont donnés dans le Tableau 6.

Dans la zone d'étude, où les réservoirs souterrains sont siliceux, les nappes ont des valeurs de $\mathrm{pH}$ faibles (5 à 6 unités $\mathrm{pH}$ ) et les eaux souterraines sont sous-saturées vis-à-vis des minéraux carbonatés (la calcite) (Sandao, 2013), on peut suggérer que les teneurs en ${ }^{13} \mathrm{C}$ déterminées sur les échantillons, sont typiques de la végétation locale, donc seulement d'origine biogénique, cela en accord avec les résultats trouvés par Ousmane (1988) et Taupin (1990). Les activités du ${ }^{14} \mathrm{C}$ déterminées sur les échantillons peuvent ainsi être utilisées sans correction par rapport aux teneurs en ${ }^{13} \mathrm{C}$.

Les valeurs des activités du ${ }^{14} \mathrm{C}$ mesurées sur les 4 échantillons prélevés varient de 37,1 à 101,9 pcm (Tableau 6), et se répartissent en 3 classes :

- l'échantillon de Mallawa, représentant les eaux de la nappe des grès argileux de Mallawa qui sont caractérisées par de faibles activités en ${ }^{14} \mathrm{C}(37,1 \%)$, résultat en accord avec l'absence du tritium dans 
ces eaux ; ce qui confirmerait le caractère essentiellement ancien de ces eaux contenues dans les grès argileux peu perméables, et dont les niveaux statiques des nappes sont les plus profonds dans le bassin de la Korama ;

- la deuxième classe, constituée des échantillons de Gouna et de Makaouratchi, représentant les eaux des nappes du socle granitique et quartzitique, avec des activités en ${ }^{14} \mathrm{C}$ relativement élevées, tout en restant inférieures à $100 \%$ pendant les périodes des hautes eaux;ce qui peut s'expliquer par l'influence de la nappe phréatique sus-jacente et l'importance des échanges entre les différents aquifères au niveau des sites; les eaux seraient à dominante récente en octobre, et ancienne en mars ;

- La $3^{\text {ème }}$ classe, avec le forage de Kantché, captant la nappe du Continental Hamadien, présente une valeur élevée de l'activité du ${ }^{14} \mathrm{C}(101,9 \mathrm{pcm})$. Ces eaux étant caractérisées par l'absence de tritium et des teneurs en ${ }^{18} \mathrm{O}$ et ${ }^{2} \mathrm{H}$ très faibles, inférieures au signal d'entrée des précipitations locales, les activités élevées en ${ }^{14} \mathrm{C}$ pourraient s'expliquer par la rémanence des gaz carboniques issus de la décomposition de la matière organique et piégée dans la zone non saturée, en milieu anaérobique.
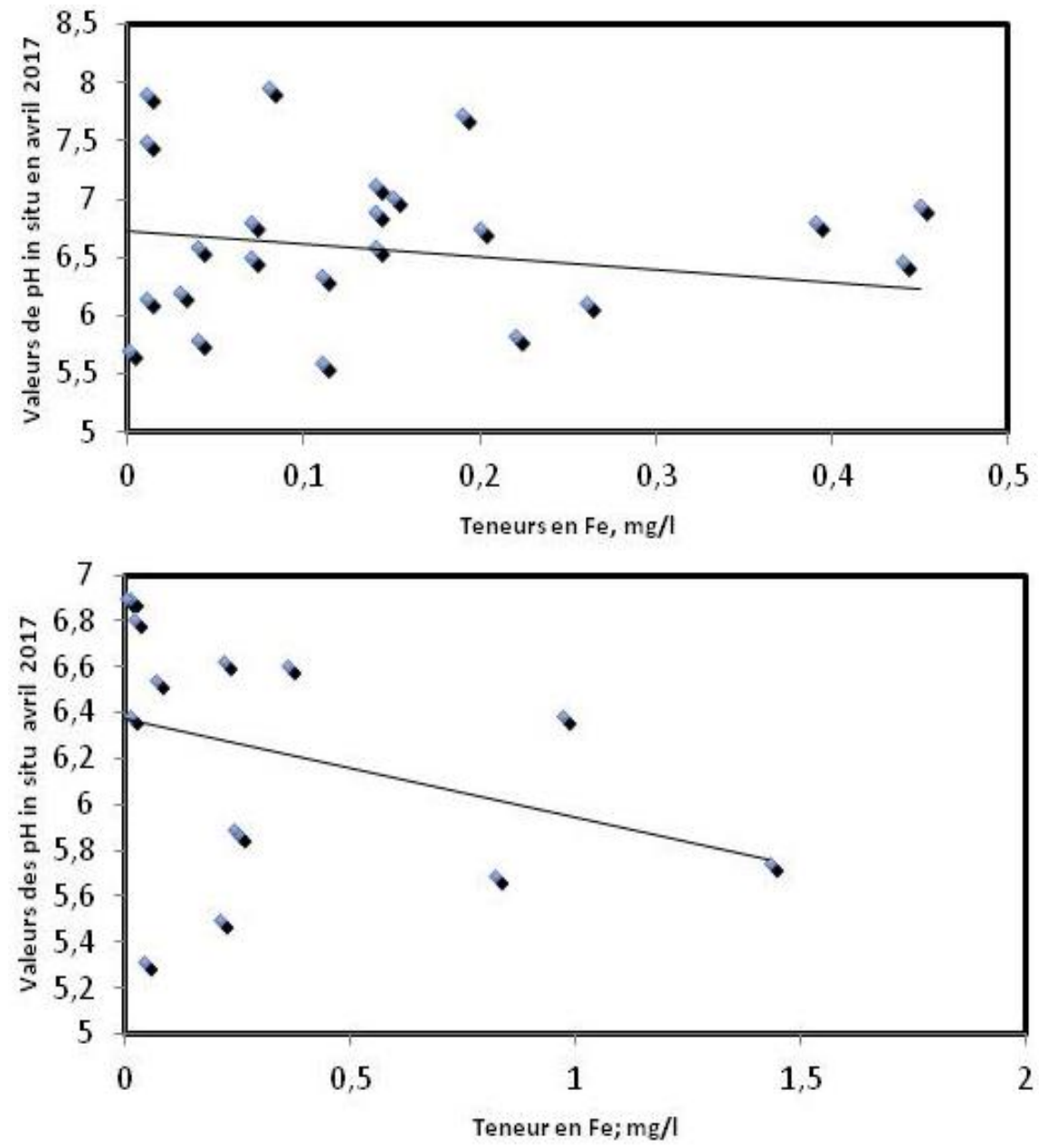

Figure 6 : Diagrammes valeurs de pH vs teneurs en Fe, pour la campagne d'avril 2017. 


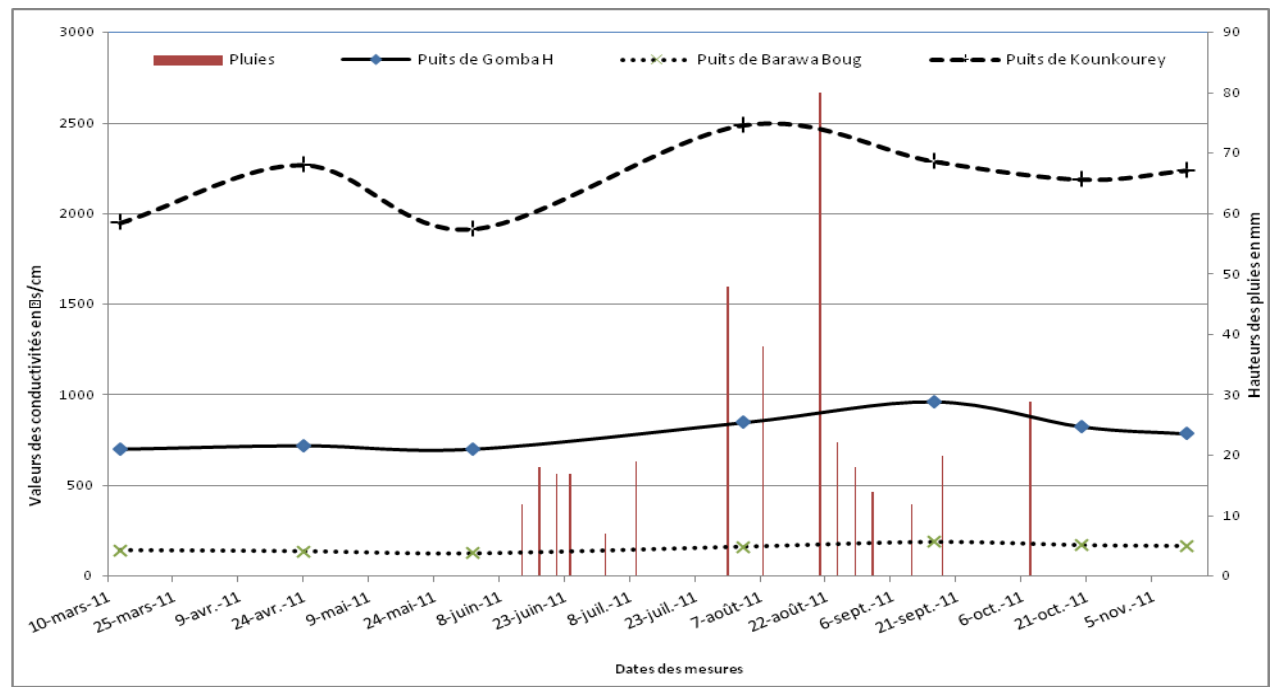

Figure 7 : Evolution saisonnière (2011) des valeurs de la minéralisation (conductivité) sur quelques sites.

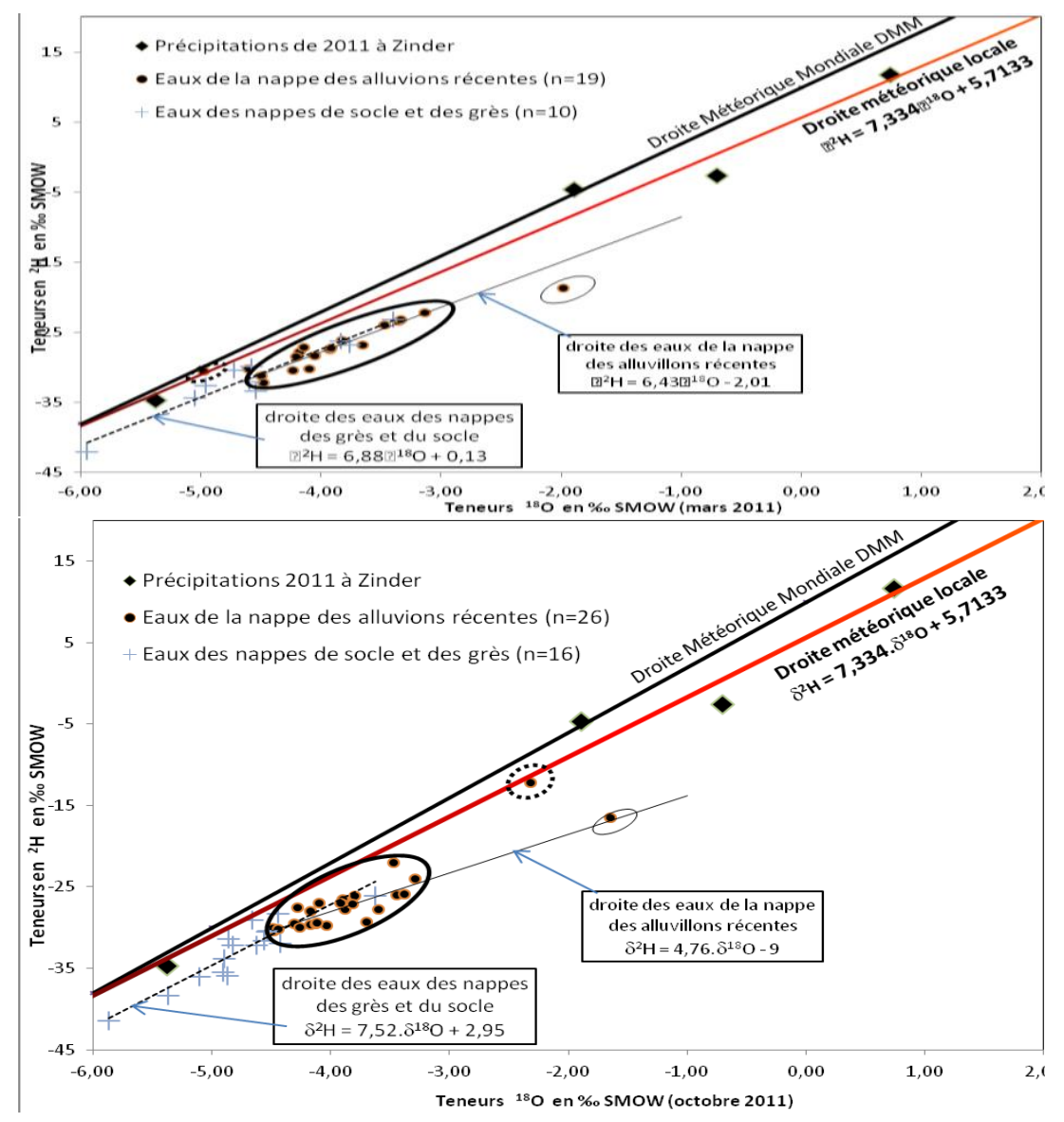

Figure 8 : Diagrammes ${ }^{2} \mathrm{Hvs}^{18} \mathrm{O}$ pour 2 campagnes de prélèvement. 


\section{Conclusion}

L'étude des paramètres hydrochimiques des eaux souterraines de la zone d'étude a permis d'établir que ces eaux sont peu minéralisées, avec des faciès chimiques bicarbonaté calcique dominants. Au niveau de certains sites captant la nappe phréatique des alluvions récentes, les eaux peuvent tendre vers le pôle nitrates-chloruressulfates. La recharge par infiltration des eaux atmosphériques est très limitée et la minéralisation des eaux serait d'origine endogène (réaction eau-roche) dans les nappes plus profondes des grès du Continental Hamadien, du Continental Terminal et des grès de Mallawa. Dans la nappe des alluvions récentes, la minéralisation est sous la dépendance des processus d'évaporation et d'infiltration des précipitations, principales sources de renouvellement de ses réserves en eau. L'analyse des paramètres isotopiques a permis de mettre en évidence que les eaux des nappes profondes des grès, à composante dominante ancienne, sont caractérisées par des taux de renouvellement quasi nuls à l'échelle locale. Elles auraient été rechargées à partir des précipitations au cours d'un paléoclimat, plus humide que l'actuel, entre 12000 et 10000 ans BP. En période des hautes eaux, la nappe phréatique dans les alluvions récentes se recharge par infiltrations des précipitations plus ou moins soumises à l'évaporation. Mais au cours de son étiage saisonnier, elle reçoit par ruissellement des eaux des autres nappes à travers les réseaux des fractures du socle et des grès continentaux.

\section{CONFLIT D'INTERETS}

Les auteurs de cet article déclarent unanimement qu'il n'y a aucun conflit d'intérêts pour cette publication.

\section{CONTRIBUTIONS DES AUTEURS}

IS a initié l'étude et a participé aux travaux de terrain (mission de suivi piézométrique et d'échantillonnage) et aux analyses dans les laboratoires; IS et MSAB ont analysé les données et ont rédigé l'article ; $\mathrm{BO}$ et JLM ont participé à la rédaction et ont fait la relecture du manuscrit.

\section{REFERENCES}

Abdou Babaye MS, Orban P, Ousmane B, Favreau G, Brouyère S, Dassargues A. 2018. Characterization of recharge mechanisms in a Precambrian basement aquifer in semi-arid south-west Niger. Hydrogeol. $J$. https://doi.org/10.1007/s10040-0181799-x.

Abdou Babaye MS, Sandao I, Saley MB, Wagani I, Ousmane B. 2016. Comportement hydrogéochimique et contamination des eaux des aquifères fissurés du socle précambrien en milieu semi-aride (Sud-Ouest du Niger). Int. J. Biol. Chem. Sci., 10(6): 2728-2743.

Abdou Babaye MS. 2012. Evaluation des ressources en eau souterraine dans le bassin de Dargol (Liptako - Niger). PhD Thesis, Univ. Liège, $235 \mathrm{p}$.

Acheamponga SY, Hess JW. 2000. Origin of the shallow groundwater system in the southern Voltaian Sedimentary Basin of Ghana: an isotopic approach. J Hydrol, 233: 37-53.

Clark I, Fritz P. 1997. Environmental Isotopes in Hydrogeology. CRC Press: Boca Raton; 328p.

DMN (Direction de la Météorologie Nationale) du Niger. 2011. Extrait de la base des données pluviométriques de la campagne 2011.

Duran A, Lang J. 1986. Approches critiques des méthodes de reconstitution paléo climatique: le Sahel nigéro-tchadien depuis 40000 ans. Bull Soc Géol France (8), 2(2) : 267-278.

Edmunds WM, Fellman E, Goni IB, Prudhomme C. 2002. Spatial and 
temporal distribution of groundwater recharge in northern Nigéria. Hydrogeol. J., 10 : 205-215.

Favreau G, Leduc C, Marlin C, Guero A. 2002. Une dépression piézométrique naturelle en hausse au Sahel (Sud-Ouest du Niger). C. R. Geoscience, 334: 395401.

Goni IB. 2006. Tracing stable isotope values from meteoric water to groundwater in the southwestern part of the Chad basin. Hydrogeol J, 14: 742-752.

GNIP. 2011. Global Network of Isotopes in Précipitation. http://nds121.iaea.org/wiser/

Greigert J. 1972. Atlas des eaux souterraines de la République du Niger. Etat des connaissances, rapport BRGM, 79 AGE001, Orléans.

Gries S. 2000. Etude géochimique et isotopique des nappes profondes au Sahara-Sahel ; implications pour la gestion des ressources en eau et les reconstitutions paléo climatiques. Thèse de doctorat Université Paris Sud, Orsay, $280 \mathrm{p}$.

Guero A. 2003. Etude des relations hydrauliques entre les différentes nappes du complexe sédimentaire de la bordure Sud-Ouest du bassin des Iullemenden (Niger): approches géochimique et hydrodynamique. Thèse de doctorat Université Paris Sud 11, Orsay, 265 p.

Guillaume F, Christian L. 2000 Représentativité de l'échantillonnage géochimique et hydrodynamique en nappe libre de milieu semi-aride. Journal of hydrology, 31(4): 669-678.

Hamza MH, Added A, France A, Rodriguez R. 2007. Validité de l'application des méthodes de vulnérabilité DRASTIC, SINTACS et SI à l'étude de la pollution par les nitrates dans la nappe phréatique de Metline-Ras Jebel-Raf Raf (Nord-Est Tunisien). C. R. Geoscience, 339 : 493505. doi:10.1016/j.crte.2007.05.003.
INS (Institut National des Statistiques du Niger). 2012. Présentation des résultats préliminaires du quatrième recensement général de la population et de l'habitat (RGP/H). Rép. du Niger, 9 p.

Lasm T, De Lasme O, Oga MSY, Youan Ta M, Baka D, Kouame F, Yao TK. 2011. Caractérisation hydrochimique des aquifères fissurés de la région de SanPedro (Sud-Ouest de la Côte d'Ivoire). Int. J. Biol. Chem. Sci., 5(2): 642-662. http://ajol.info/index.php/ijbcs.

Maliki A, Krimissa M, Michelot JL, Zouari K. 2000. Pas cité dans le texte. Relation entre nappes superficielles et aquifère profond dans le bassin de Sfax (Tunisie). C. R. Acad. Sci. Paris, Sciences de la Terre et des planètes / Earth and Planetary Sciences, 331: 1-6.

Ousmane B. 1988. Etude géochimique et isotopique des aquifères du socle de la bande sahélienne du Niger (Liptako, Sud Maradi et Zinder Est), Thèse de doctorat d'état, Université de Niamey. 175 p.

Sandao I. 2013. Etude hydrodynamique, Hydrochimique et isotopique des eaux souterraines du bassin de la Korama: Sud Zinder, Niger: Impact de la variabilité climatique et des activités anthropiques. Thèse de doctorat, Université de Niamey, $240 \mathrm{p}$.

Sandao I. 2010. Contribution à la connaissance de l'état actuel des ressources en eau du bassin de la Korama: Sud de la Région de Zinder, mémoire de DEA, Université de Niamey, $96 \mathrm{p}$.

Stadler S, Osenbrück K., Suckow AO, Himmelsbach T, Hötzl H. 2010. Groundwater flow regime, recharge and regional-scale solute transport in the semi-arid Kalahari of Botswana derived from isotope hydrology and hydrochemistry. J. Hydrol., 388 : 291303. 
SOGETHA. 1964. Les Koramas: reconnaissance hydrogéologique, annexes, figures et cartes. Ministère de l'Economie Rurale du Niger. 50 p.

Soumaila IH. 2016. Evaluation qualitative et quantitative des ressources en eau de la nappe phréatique de la Korama (Sud Zinder). Mémoire de Master, Université Abdou Moumouni de Niamey, FAST, 95 p.

Taupin JD 1994. Utilisation des méthodes isotopiques dans l'étude de différentes parties du cycle de l'eau dans le cadre d'HAPEX-SAHEL; $10^{\text {ème }}$ journées hydrologiques ORSTOM - septembre 1994, pp 335-352.

Tremoy G, Vimeux F, Mayaki S, Souley I, Cattani O, Risi C, Favreau G, Oï M. 2012. A 1-year long d 180 record of water vapor in Niamey (Niger) reveals insightful atmospheric processes at different timescales. Geophysical Research Letters, 39: L08805. Doi: 10.1029/2012GL051298, 5p.

Zakara Z. 1990. Contribution à l'étude hydrogéologique des nappes des Korama Zinder République du Niger, mémoire de diplôme de 3ème cycle du Centre d'Hydrogéologie, Université de Neuchâtel 90 p.

Zakara Z, Atahirou K, Aranyossy F. 1993. Application des isotopes de l'environnement à l'étude des aquifères des Korama, sud de Zinder (Niger). Comptes rendus des études effectuées dans le cadre du projet RAF/8/012: Hydrologie isotopique dans les pays du Sahel. Niamey, Niger. $10 \mathrm{p}$. 